\title{
AN EXAMINATION OF THE EFFECTS OF THE CRITERION FUNCTIONAL ON OPTIMAL FIRE-SUPPORT POLICIES*
}

\author{
James G. Taylor and Gerald G. Brown \\ Naval Posstgraduate School \\ Monterey, California
}

\begin{abstract}
This paper examines the dependence of the structure of optimal time-sequential fire-support policies on the quantification of military objectives by considering four specific problems, each corresponding to a different quantification of objectives (i.e. criterion functional). We consider the optimal time-sequential allocation of supporting fires during the "approach to contact" of friendly infantry against enemy defensive positions. The combat dynamics are modelled by deterministic Lanchester-type equations of warfare, and the optimal fire-support policy for each one-sided combat optimization problem is developed via optimal control theory. The problems are all nonconvex, and local optima are a particular difficulty in one of them. For the same combat dynamics, the splitting of supporting files between twio enemy forces in any optimal policy (i.e. the optimality of singular subarcs) is shown to depend only on whether the terminal payoff reflects the objective of attaining an "overall" military advantage or a "local" one. Additionally, switching times for changes in the ranking of target priorities are shown to be different (sometimes significantly) when the decision criterion is the difference and the ratio of the military worths (computed according to linear utilities) of total infantry survivors and also the difference and the ratio of the military worths of the combatants' total infantry losses. Thus, the optimal fire-support policy for this attack scenario is shown to be significantly influenced by the quantification of military objectives.
\end{abstract}

\section{INTRODUCTION}

As one of the authors has pointed out in [40], for the purposes of military operations research it is convenient to consider that there are three essential parts of any time-sequential combat optimization problem:

(a) the decision criteria (for both combatants),

(b) the model of conflict termination (and/or unit breakpoints),

(c) the model of combat dynamics.

An important problem of military operations research is the determination of the relationship between the nature of system objectives and the structure of optimal combat strategies. Of particu-

*This research was partially supported by the Office of Naval Research. The authors wish to thank the referee for his helpful suggestions. A slightly expanded version of this paper (with numerous annotations elaborating upon various points) has appeared in report form as [45]. 
lar importance is the sensitivity of the structure of optimal combat strategies to the nature of military objectives (see [29] for a discussion of the influences of political objectives on military objectives for the evaluation of (timesequential) combat strategies). In a time-sequential combat optimization problem the combatant objectives are quantified through the criterion functional [8]. If the optimal combat strategy and associated payoff are quite sensitive to the functional form of the criterion functional, then care must be exercised in the selection of the functional form.

An important constituent part of fire support is the target allocation function which matches a specific weapon type with an acquired target within the target's environment [25]. It is not surprising then that the determination of optimal target allocation strageties for supporting weapon systems [48] is (in one form or another) one of the most extensively studied problems in both the open literature $[42,43]$ and also classified sources. During World War II the problem of the appropriate mixture of tactical and strategic air forces (another aspect of the optimal fire-support strategy problem) was extensively debated by experts. Some analysis details are to be found in the classic book by Morse and Kimball (see pp. 73-77 of [27]). This problem was further studied at RAND in the late 1940's and early 1950's [13] and elsewhere [3]. It would probably not be too farfetched to say that this problem stimulated early research on both dynamic programming [4] and also differential games $[13,18]$. Today the problem of the determination of optimal air-war strategies (another aspect of the fire-support problem) is being rather extensively studied by a number of organizations $[1,2,5,6,10,12,14,22,46]$.

Thus, the objective of this investigation is to determine the sensitivity of the optimal timesequential fire-support policy to the functional form of the criterion functional. Our research approach is to combine Lanchester-type models of warfare (see, for example, [34, 38, 40] and references contained therein) with generalized control theory [15, 16] (i.e. optimization theory for dynamic systems). This general research program has been described in more detail elsewhere $[40,41]$. It seems appropriate to examine sensitivity of the optimal policy by considering a concrete problem. Consequently, our research approach is to consider several different criterion functionals for the same tactical situation involving the allocation of supporting fires. The tactical situation that we have chosen to examine is the "approach to contact" during an assault on enemy defensive positions by friendly ground forces. We seek to determine the "best" allocation for the supporting fires of the friendly forces. Weiss [48] has emphasized that a simplified model of a combat situation is particularly valuable when it leads to a clearer understanding of significant relationships which would tend to be obscured in a more complex model. Consequently, we will consider a mathematically tractable version of this problem so that we can make quantitative comparisons among the optimal policies corresponding to the various criterion functionals. Corresponding to each different criterion functional is a different optimization (here, optimal control) problem. Each of these problems has been solved, and the corresponding optimal fire-support policies will be contrasted.

In this paper four different criterion functionals are considered: it is shown that both the difference and the ratio of military worths of friendly and enemy survivors (computed according to linear utilities) and also the ratio of the military worths of friendly and enemy losses as criterion functionals may lead to exactly the same optimal policy. A completely different optimal policy, however, is obtained for the weighted average of force ratios of opposing infantry (at the time that the supporting fires are lifted) as the criterion functional. We have decided that the three former criterion functionals (i.e. the difference and the ratio of the military worths of survivors and the 
ratio of the military worths of losses) are appropriate for an "attrition" objective,* whereas the weighted average of force ratios is appropriate for a "breakthrough" objective. $\dagger$ (In the latter case, the attacking force tries to overpower the defenders at one place along a front and then pour reinforcements through the break in the defender's defenses in order to "penetrate" behind the enemy lines and, for example, disrupt enemy command, control, and communications.)

The body of this paper is organized in the following fashion. First, we review previous work on the relationship between the quantification of military objectives and the structure of optimal time-sequential fire-distribution policies in order to place the work at hand in proper perspective. Then we describe the fire-support problem and discuss the four criterion functionals that will be used to determine optimal fire-support policies. Each of these criterion functionals represents a different quantification of military objectives, and all appear to be reasonable criteria. Next, the optimal fire-support policies are described for the four problems. The structures of the four optimal policies are then contrasted. Next, we justify the optimization results that we have been discussing by sketching their development via modern optimal control theory. This development is given for each of the four problems. Finally, we discuss what we have learned from our investigation of the dependence of the structure of optimal fire-support policies on the quantification of military objectives.

\section{PREVIOUS WORK ON THE STRUCTURE OF OPTIMAL FIRE-DISTRIBUTION POLICIES}

The only systematic examinations of the influences of the nature of the criterion function on the structure of optimal time-sequential fire-distribution strategies known to the authors are those of Taylor [30-33, 35, 37, 40,43]. In [31] and [40], however, the influences of the nature of the targettype attrition process on the structure of optimal fire-distribution policies were examined. In [30-33] and [40] a linear utility $\$$ was assumed for the military worth of the number of each surviving weapon system type, and the criterion functional (payoff) was taken to be the net military worth of survivors (i.e. the difference between the military worths of friendly and enemy forces). Taylor $[30-33,40]$ has studied how the optimal fire-distribution policy depends on the assignment of these linear utilities. In other words, he examined the sensitivity of the optimal combat policy to parametric variations in the assigned linear utilities for survivors. It has been shown that the $n$-versusone fire-distribution problems studied in [30-33] all have quite simple solutions when enemy survivors are valued in direct proportion to their kill capabilities (as measured by their Lanchester attrition-rate coefficients [34,38] against the (homogeneous) friendly forces).

Pugh and Mayberry [29] have suggested that a appropriate payoff, or objective function (in our terminology, criterion functional) for the quantitative evaluation of combat strategies is the loss ratio (calculated possibly using weighting factors for heterogeneous forces). They have stated

*In other words, the friendly forces seek an "overall" military advantage.

$\dagger$ In other words, the friendly forces seek a "local" military advantage.

¥ See [17] for the methodology for the development of these linear utilities. For optimal control differential game combat optimization problems, the assumption of linear utilities yields that the boundary conditions for the adjointvariables (at least when no terminal state constraint is active) are independent of the values of the state variables. Serious computational difficulties may arise when nonlinear utilities are assumed. The effects of assuming nonlinear utilities for military resources upon the evaluation of time-sequential combat strategies has apparently never been studied. 
[29] that an "almost equivalent" criterion is the loss difference. However, Pugh and Mayberry [29] do not explore the consequences of various functional forms for the criterion functional. In this paper we will examine to what extent these criteria are, in fact, equivalent. In combat problems with either no replacements or a fixed-length planning horizon, it is readily seen that minimizing the loss difference is the same as maximizing the difference in survivors. It is such a case of no replacements that we will examine here. It remains to determine the "equivalence" of minimizing the loss ratio to maximizing the ratio of survivors and to relate these results to those for maximizing the difference in survivors.

Furthermore, for the evaluation of combat strategies it is of interest to consider the military worth (i.e. utility of military resources) of survivors. In almost all* the work that has appeared in the open literature [41], a linear utility has been assumed for valuation of survivors, and some form of net military worth (i.e. the difference between the military worths of friendly and enemy survivors) has been taken as the payoff (i.e. criterion functional) [26, 30-33, 35, 40,41]. One reason for assuming such linear utilities is that of mathematical tractability: the boundary conditions for the dual variables do not depend on the state variable values (at least when no terminal constraint involving the state variables is active).

The only study known to the authors of the consequences on nonlinear utilities for survivors is contained in [37], where Kawara's supporting weapon system game [20] is examined. Taylor [37] has determined (at least for the case in which the appropriate side's (in Kawara's case, the defender) supporting weapon system is not annihilated) the most general form of the criterion functional which leads to optimal fire-support strategies being independent of force levels, and he has shown that the criterion functional chosen by Kawara [20] is a special case of this form. In other words, Taylor has shown that Kawara's conclusion [20] that optimal fire-support strategies do not depend on force levels only applies to problems with the special type of criterion functional used by Kawara and is not true in general. No other examination of the dependence of optimal combat strategies on combatant objectives is known to the authors.

\section{COMPARISON OF OPTIMAL FIRE-SUPPORT POLICIES}

In this section we give the fire-support allocation problem for which the optimal policy is developed according to four different criterion functions. These fire-support policies are then compared.

\subsection{The Fire-Support Problem}

Let us consider the attack of heterogeneous $X$ forces against the static defense of heterogeneous $Y$ forces along a "front." Each side is composed of primary units (or infantry) and fire-support units (or artillery). The $X$ infantry (denoted as $X_{1}$ and $X_{2}$ ) launches an attack against the positions held by the $Y$ infantry (denoted as $Y_{1}$ and $Y_{2}$ ). We may consider $X_{1}$ and $X_{2}$ to be infantry units

*The only exceptions known to the authors are the papers by Chattopadhyay [9] and Kawara [20]. For example, in Kawara's paper [20] the payoff is the ratio of opposing infantry strengths (measured in terms of total numbers) at the "end of battle" (see also the differential game studied in Appendix D of [43]). 
operating on spatially separated pieces of terrain. We assume that the $X_{1}$ infantry unit attacks the $Y_{1}$ infantry unit and similarly for $X_{2}$ and $Y_{2}$ with no "crossfire." (e.g. the $X_{1}$ infantry is not attrited by the $Y_{2}$ infantry). We will consider only the "approach to contact" phase of the battle. This is the time from the initiation of the advance of the $X_{1}$ and $X_{2}$ forces towards the $Y_{1}$ and $Y_{2}$ defensive positions until the $X_{1}$ and $X_{2}$ forces actually make contact with the-enemy infantry in "hand-to-hand" combat. It is assumed that this time is fixed and known to $X$.

The $X_{i}$ forces begin their advance against the $Y_{i}$ forces from a distance and move towards the $Y_{i}$ position. The objective of the $X_{i}$ forces during the "approach to contact" is to close with the enemy position as rapidly as possible. Accordingly, small arms fire by the $X_{i}$ forces is held at a minimum or firing is done "on the move" to facilitate rapid movement. It is not unreasonable, therefore, to assume that the effectiveness of $X_{i}$ force "on the move" is negligible against $Y_{\imath}$. It may be shown that such an approximation is necessary for reasons of mathematical tractability in the fire-support optimal control problem to be subsequently given. See the Appendix for further details. We assume, moreover, that the defensive $Y_{i}$ fire (for $i=1,2$ ) causes attrition to the advancing $X_{i}$ forces in their "field of fire" at a rate proportional to only the number of $Y_{i}$ firers. Let $a_{i}$ denote the constant of proportionality. It is convenient to refer to the attrition of a target type as being a "square-law" process when the casualty rate is proportional to the number of enemy firers only and as being a "linear-law" process when it is proportional to the product of the number of enemy firers and remaining targets [31-33]. Brackney [7] has hypothesized that a "square-law" attrition process occurs when the time to acquire targets is negligible in comparison with the time to destroy them. He has pointed out that such a situation is to be expected to occur when one force assaults another. Additionally, we assume that either the $Y$ forces have no fire-support units or their fire support is "organic" to the $Y$ units (i.e. fire-support units are integrated with $Y_{i}$ and only those with $Y_{i}$ support $Y_{i}$ ).

During the "approach to contact" the $X$ fire-support units (denoted as $W$ ) deliver "area fire" against the $Y_{i}$ forces. In other words, we assume that $X$ 's fire-support units fire into the (constant) area containing the enemy's infantry without feedback as to the destructiveness of this fire. Let $\phi_{i}$ denote the fraction of the $W$ fire-support units which fire at $Y_{i}$. (We then have that $\phi_{1}+\phi_{2}=1$ and $\phi_{i} \geq 0$ for $i=1,2$.) Then for constant $\phi_{i}$ there are a constant number of fire-support units firing at $Y_{i}$, since we assume that the $W$ fire-support units are not in the combat zone and do not suffer attrition. In this case, the $Y_{i}$ attrition rate is proportional to the $Y_{i}$ force level $[19,47]$. Let $c_{i}$ denote the corresponding constant of proportionality. This combat situation is shown diagrammatically in Figure 1.

It is the objective of the $X$ forces to utilize their fire-support units (denoted as $W$ ) over time in such a manner so as to achieve the "most favorable" situation at the end of the "approach to contact", at which time the force separations between opposing infantries are zero and artillery fires must be lifted from the enemy's positions in order not to also kill friendly forces. The "outcome" of this phase of battle may be measured in several different ways and is quantitatively expressed through the criterion functional (denoted as $J$ ). Thus, we have the following optimal control problem for the determination of the optimal fire-support allocation policy (denoted as 


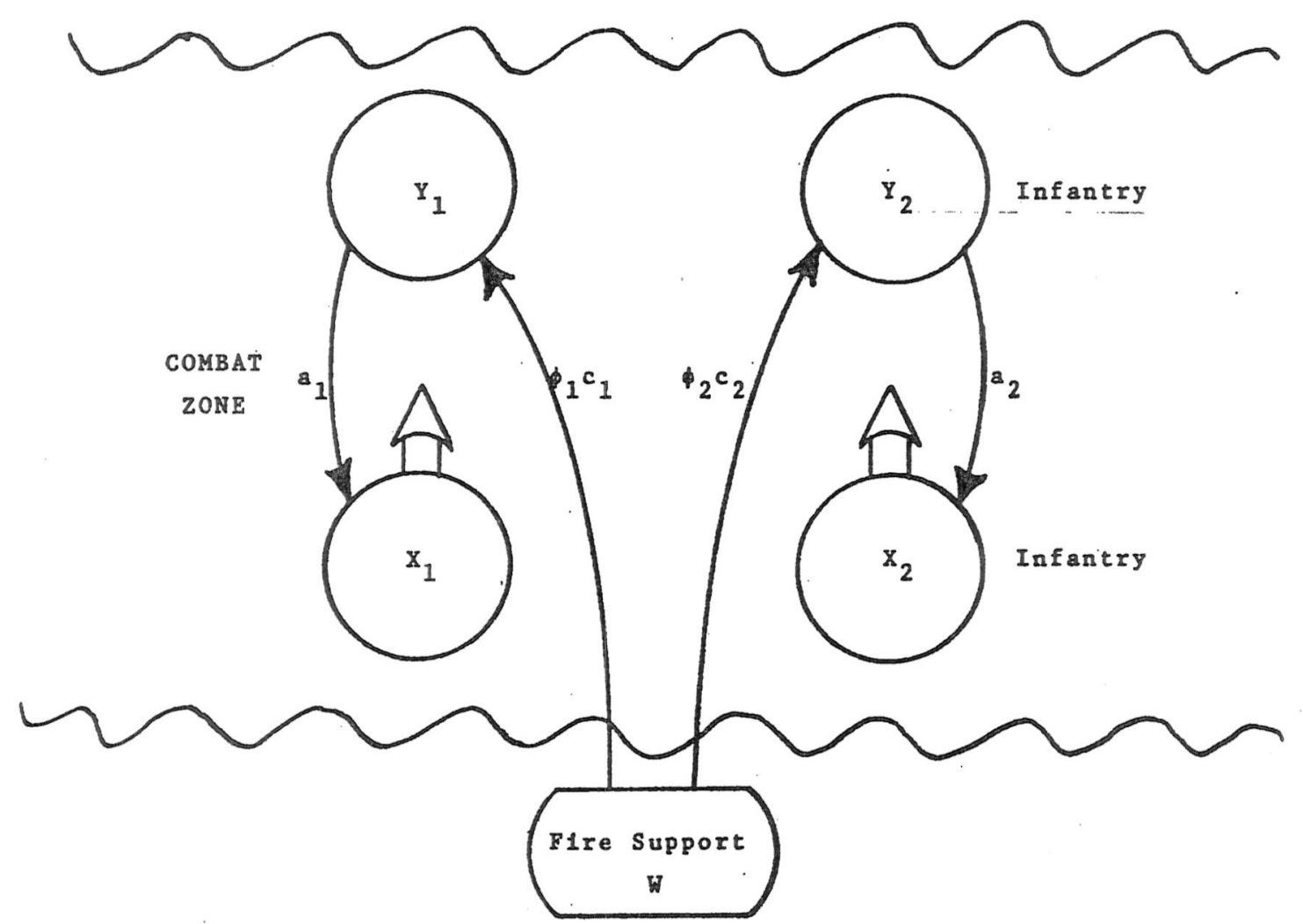

FIGURE 1. Diagram of fire-support problem considered for examination of effect of criterion functional on optimal fire-support policy.

$\phi^{*}(t)$ for $0 \leq t \leq T$, where $T$ denotes the time of the end of the "approach to contact") for the $W$ fire-support units:

$$
\underset{\phi_{i}(t)}{\operatorname{maximize}} J
$$

with stopping rule: $t_{\rho}-T=0$,

$$
\begin{array}{ll}
\begin{array}{ll}
\text { subject to: } \\
\text { (battle dynamics) }
\end{array} & \frac{d x_{i}}{d t}=-a_{i} y_{i}, \\
& \frac{d y_{i}}{d t}=-\phi_{i} c_{i} y_{i} \quad \text { for } i=1,2,
\end{array}
$$

with initial conditions:

and

$$
x_{i}(0)=x_{i}{ }^{0} \text { and } y_{i}(0)=y_{. i}{ }^{0} \quad \text { for } i=1,2,
$$

$x_{1}, x_{2} y_{1}, y_{2} \geq 0$ (State Variable Inequality Constraints),

where

$$
\phi_{1}+\phi_{2}=1 \text { and } \phi_{i} \geq 0 \text { for } i=1,2 \text { (Control Variable Inequality Constraints), }
$$

$J$ denotes the criterion functional,

$x_{i}(t)$ denotes the number of $X_{i}$ infantry at time $t$, similarly for $y_{i}(t)$, 
$a_{i}$ is a constant (Lanchester) attrition-rate coefficient (reflecting the effectiveness of $Y_{i}$ fire against $X_{i}$ ),

$c_{i}$ is a constant (Lanchester) attrition-rate coefficient (reflecting the effectiveness of $W$ supporting fires against $Y_{i}$ ),

$t_{f}$ (with numerical value $T$ ) denotes the end of the optimal control problem, and

$\phi_{i} \quad$ denotes the fraction of $W$ fire support directed at $Y_{i}$.

It will be convenient to consider the single control variable $\phi$ defined by

$$
\phi=\phi_{1} \text { so that } \phi_{2}=(1-\phi) \text { and } 0 \leq \phi \leq 1 \text {. }
$$

For $T<+\infty$ it follows that $y_{i}(t)>0$ for $0 \leq t \leq T$. Thus, the only state variable inequality constraints (SVIC's) that must be considered are $x_{i} \geq 0$. However, let us further assume that the attacker's infantry force levels are never reduced to zero. This assumption applies to all feasible solutions and may be militarily justified on the grounds that $X$ would not attack the $Y_{1}$ positions if his attacking $X_{i}$ forces could not survive the "approach to contact."

\subsection{The Criterion Functionals Considered}

The four criterion functionals for which the optimal fire-support allocation policies will be compared are given in Table 1. All are functions only of the various numbers of combatants at the end of the planning horizon (i.e. at the end of the "approach to contact" at which time the supporting fires must be lifted for safety reasons).

TABLE I. Summary of problems considered to study effect of criterion functional on optimal firesupport policy.

\begin{tabular}{|c||c|}
\hline Problem & Criterion Functional, $J$ \\
\hline 1 & $\frac{\sum_{k=1}^{2} \alpha_{k} x_{k}(T) / y_{k}(T)}{\sum_{k=1}^{2} v_{k} x_{k}(T)-\sum_{k=1}^{2} w_{k} y_{k}(T)}$ \\
\hline 3 & $-\left\{\sum_{k=1}^{2} v_{k} x_{k}(T)\right\} /\left\{\sum_{k=1}^{2} w_{k} y_{k}(T)\right\}$ \\
\hline 4 & $-\left\{\sum_{k=1}^{2} v_{k}\left(x_{k}^{0}-x_{k}(T)\right)\right\} /\left\{\sum_{k=1}^{2} w_{k}\left(y_{k}^{0}-y_{k}(T)\right)\right\}$ \\
\hline
\end{tabular}

The criterion functional for Problem 1 (i.e. $J_{1}=\sum_{k=1}^{2} \alpha_{k} x_{k}(T) / y_{k}(T)$ ) represents a weighted a verage of the force ratios of opposing numbers of infantry in the two infantry combat zones. The rationale behind this choice is that, in each combat area, (i.e. the area of combat between $X_{1}$ and $Y_{i}$ ) combat (possibly hand-to-hand) between the $X_{i}$ and $Y_{i}$ forces will follow the "approach to contact" and the (initial) force ratio will be related to the outcome of this subsequent combat 
action. The weighting factors (i.e. $\alpha_{k}$ for $k=1,2$ ) allow one to assign relative weights to this subsequent combat between $X_{i}$ and $Y_{i}$ in the two combat areas.

The criterion functional for Problem 2 (i.e. $J_{2}=\Sigma_{k=1}^{2} v_{k} x_{k}(T)-\Sigma_{k=1}^{2} w_{k} y_{k}(T)$ ) represents the difference between the military worths (computed using linear utilities) of the surviving $X$ and $Y$ forces at the end of the "approach to contact." As noted above in Section 2, we observe that maximizing the difference in worth of survivors is the same as minimizing the loss difference in combat problems (such as the one at hand) with no replacements. The criterion functional for Problem 3 (i.e. $J_{3}=\left\{\Sigma_{k=1}^{2} v_{k} x_{k}(T)\right\} /\left\{\Sigma_{k=1}^{2} w_{k} y_{k}(T)\right\}$ ) represents the ratio of total military worths of the surviving $X$ and $Y$ forces, whereas the one for Problem 4 (i.e. $J_{4}=-\left\{\Sigma_{k=1}^{2} v_{k}\left(x_{k}{ }^{0}-x_{k}(T)\right)\right\}$ / $\left.\left\{\Sigma_{k=1}^{2} w_{k}\left(y_{k}^{0}-y_{k}\left(T^{T}\right)\right)\right\}\right)$ represents the ratio of military worths of losses. Both the loss ratio and the loss difference have been proposed by Pugh and Mayberry [29] as appropriate payoffs for the evaluation of combat strategies. They state that (see p. 869 of [29]) "when the most straightforward estimate of a weighting factor for the loss difference is used, the two criteria are almost equivalent." From the study at hand, we will see that a similar statement is true: the two criteria are equivalent for a certain "natural" valuation of forces (see next section), but otherwise they may yield slightly different optimal fire-support policies.

\subsection{Optimal Fire-Support Policies}

In this section we give the optimal time-sequential fire-support policies for the four problems presented in the previous section. As discussed above, each of these problems corresponds to a different decision criterion for the attackers, with all other aspects of the problem (i.e. combat dynamics and length of the planning horizon) being the same in all problems. In all cases we assume that neither of the attacking infantry forces can be reduced to a zero force level during the approach to contact, i.e. problem parameters and initial force levels are such that $x_{i}(T)>0$ for $i=1,2$.

For Problem 1 with $J_{1}=\Sigma_{k=1}^{2} \alpha_{k} x_{k}(T) / y_{k}(T)$, the optimal (open-loop) fire-support policy is

$$
\phi^{*}\left(t ; r_{1}^{0}, r_{2}^{0}, T\right)=\left\{\begin{array}{l}
1 \text { for } 0 \leq t \leq T \text { when } F_{1}\left(r_{1}^{0}, T\right) \geq F_{2}\left(r_{2}{ }^{0}, T\right), \\
0 \text { for } 0 \leq t \leq T \text { when } F_{1}\left(r_{1}^{0}, T\right) \leq F_{2}\left(r_{2}^{0}, T\right),
\end{array}\right.
$$

where $r_{i}(t)=r_{i}=x_{i} / y_{i}, r_{i}{ }^{0}=r_{i}(0)$, and

$$
F_{i}\left(r_{i}^{0}, T\right)=\alpha_{i} a_{i} c_{i}\left\{\left(\frac{r_{i}{ }^{0}}{a_{i}}\right)\left(\frac{e^{c_{i} T}-1}{c_{i}}\right)-\frac{1}{c_{i}^{2}}\left(e^{c_{i} T}-1-c_{i} T\right)\right\} .
$$

For problem 2 with $J_{2}=\Sigma_{k=1}^{2} v_{k} x_{k}(T)-\Sigma_{k=1}^{2} w_{k} y_{k}(T)$ and Problem 3 with $J_{3}=\left\{\Sigma_{k=1}^{2} v_{k} x_{k}(T)\right\} /$ $\left\{\Sigma_{k=1}^{2} w_{k} y_{k}(T)\right\}$, we make the nonrestrictive assumption that

$$
\frac{w_{1}}{a_{1} v_{1}} \geq \frac{w_{2}}{a_{2} v_{2}}
$$

Then the optimal (closed-loop) fire-support policy may be expressed in the same form for Problems 2 and 3 . It is best explained by considering that the battle is divided into two phases, denoted as Phase I and Phase II. During Phase I for $0 \leq t<t_{1}=T-\tau_{1}\left(y_{1}{ }^{\gamma} / y_{2}\right)$, the optimal policy is

$$
\phi^{*}(t, x, y)= \begin{cases}1 & \text { for } \rho>a_{2} c_{2} v_{2} /\left(a_{1} c_{1} v_{1}\right), \\ c_{2} /\left(c_{1}+c_{2}\right) & \text { for } \rho=a_{2} c_{2} v_{2} /\left(a_{1} c_{1} v_{1}\right), \\ 0 & \text { for } \rho<a_{2} c_{2} v_{2} /\left(a_{1} c_{1} v_{1}\right),\end{cases}
$$


where

$$
\rho=y_{1} / y_{2}
$$

while during Phase II for $T-\tau_{1}\left(y_{1}{ }^{f} / y_{2}{ }^{f}\right) \leq t \leq T$, the optimal policy is

$$
\phi^{*}(t, x, y)=1
$$

where

$$
\begin{gathered}
\tau_{1}=\tau_{1}\left(y_{1}^{f} / y_{2}^{f}\right)=\left\{\begin{array}{llr}
\tau_{S} & \text { for } & \rho^{f} \geq \rho_{S}^{f} \\
\tau_{\phi} & \text { for } \rho_{L} \leq \rho^{f}<\rho_{S}^{f} \\
0 & \text { for } & \rho^{f}<\rho_{L}
\end{array}\right. \\
\rho_{L}=\left(\frac{a_{2} c_{2} v_{2}}{a_{1} c_{1} v_{1}}\right)\left(\frac{w_{2}}{a_{2} v_{2}}\right) /\left(\frac{w_{1}}{a_{1} v_{1}}\right)
\end{gathered}
$$

and $\rho_{S}{ }^{f}$ denotes the final ratio $\left(y_{1} / y_{2}\right)^{f}$ such that as one works backwards from this end point, the optimal path leads "directly to" the "singular surface" after requiring use of the policy (8) for a finite interval of time. We will examine below how $\tau_{1}$ depends on whether or not inequality holds in (5). Furthermore, $\tau_{S}$ is the unique non-negative root of $F\left(\tau_{S}\right)=0$, where the function $F(\tau)$ is different for Problems 2 and 3 and is given below. For $\rho_{L}<\rho^{f}<\rho_{s}{ }^{f}, \tau_{\phi}$ is the smaller of the two positive roots of $G\left(\tau_{\phi} ; \rho^{f}\right)=0$, where the function $G\left(\tau ; \rho^{f}\right)$ is also different for Problems 2 and 3 and is given below. $\tau_{S}$ and $\tau_{\phi}$ may be called switching times. It has been shown that

(a) bounds on $\tau_{\phi}$ are given by $0 \leq \tau_{\phi}<\tau_{S}$,

(b) $\tau_{\phi}$ is a strictly increasing function of $\rho^{f}$ for $\rho_{L} \leq \rho^{f}<\rho_{S}{ }^{f}$, and

(c) there is no root to $G\left(\tau_{\phi} ; \rho^{f}\right)$ for $\rho^{f}>\rho_{s}{ }^{j}$.

For Problem 2, we have

$$
\begin{gathered}
F(\tau)=\tau+\left(\frac{1}{c_{1}}-\frac{w_{1}}{a_{1} v_{1}}\right) e^{-c_{1 \tau}}-\left(\frac{1}{c_{1}}-\frac{w_{2}}{a_{2} v_{2}}\right), \text { and } \\
G\left(\tau ; \rho^{f}\right)=\frac{1}{c_{1}}\left(e^{c_{1 \tau}}-1\right)\left(\frac{a_{1} c_{1} v_{1}}{a_{2} c_{2} v_{2}}\right) \rho^{\rho}-\tau+\left(\frac{a_{1} c_{1} v_{1}}{a_{2} c_{2} v_{2}}\right)\left(\frac{w_{1}}{a_{1} v_{1}}\right) \rho^{\rho}-\left(\frac{w_{2}}{a_{2} v_{2}}\right) .
\end{gathered}
$$

Bounds on $\tau_{S}$ in Problem 2 are given by (a) for $w_{1} /\left(a_{1} v_{1}\right) \leq 1 / c_{1}$,

$$
\frac{w_{1}}{a_{1} v_{1}}-\frac{w_{2}}{a_{2} v_{2}} \leq \tau_{S} \leq \frac{1}{c_{1}}\left\{1-\left(\frac{w_{2}}{a_{2} v_{2}}\right) /\left(\frac{w_{1}}{a_{1} v_{1}}\right)\right\}, \text { and }
$$

(b) for $1 / c_{1} \leq w_{1} /\left(a_{1} v_{1}\right)$,

$$
\frac{1}{c_{1}}\left\{1-\left(\frac{w_{2}}{a_{2} v_{2}}\right) /\left(\frac{w_{1}}{a_{1} v_{1}}\right)\right\} \leq \tau_{S} \leq \frac{w_{1}}{a_{1} v_{1}}-\frac{w_{2}}{a_{2} v_{2}}
$$

For Problem 3, we have

$$
\begin{gathered}
F(\tau)=\tau+\left(\frac{1}{c_{1}}-\frac{J_{3} w_{1}}{a_{1} v_{1}}\right) e^{-c_{1} \tau}-\left(\frac{1}{c_{1}}-\frac{J_{3} w_{2}}{a_{2} v_{2}}\right), \text { and } \\
G\left(\tau ; \rho^{\gamma}\right)=\frac{1}{c_{1}}\left(e^{c_{1} \tau}-1\right)\left(\frac{a_{1} c_{1} v_{1}}{a_{2} c_{2} v_{2}}\right) \rho^{f}-\tau+J_{3}\left\{\left(\frac{a_{1} c_{1} v_{1}}{a_{2} c_{2} v_{2}}\right)\left(\frac{w_{1}}{a_{1} v_{1}}\right) \rho^{f}-\left(\frac{w_{2}}{a_{2} v_{2}}\right)\right\} .
\end{gathered}
$$

Bounds on $\tau_{S}$ in Problem 3 are given by (a) for $J_{.3} w_{1} /\left(a_{1} v_{1}\right) \leq 1 / c_{1}$, 


$$
J_{3}\left(\frac{w_{1}}{a_{1} v_{1}}-\frac{w_{2}}{a_{2} v_{2}}\right) \leq \tau_{S} \leq \frac{1}{c_{1}}\left\{1-\left(\frac{w_{2}}{a_{2} v_{2}}\right) /\left(\frac{w_{1}}{a_{1} v_{1}}\right)\right\}, \text { and }
$$

(b) for $1 / c_{1} \leq J_{3} w_{1} /\left(a_{1} v_{1}\right)$,

$$
\frac{1}{c_{1}}\left\{1-\left(\frac{w_{2}}{a_{2} v_{2}}\right) /\left(\frac{w_{1}}{a_{1} v_{1}}\right)\right\} \leq \tau_{S} \leq J_{3}\left(\frac{w_{1}}{a_{1} v_{1}}-\frac{w_{2}}{a_{2} v_{2}}\right) .
$$

Also for Problem 3, we have

$$
\partial \tau_{S} / \partial J_{3}>0
$$

The solution to Problem 4 is exactly like that to Problem 3 except that $J_{3}$ in Problem 3 is replaced by $\left(-J_{4}\right)$.

Let us now sketch the proofs of a few statements just made above about the switching times $\tau_{S}$ and $\tau \phi$. The existence of a unique nonnegative root to $F\left(\tau_{S}\right)=0$ for $w_{1} /\left(a_{1} v_{1}\right) \geq w_{2} /\left(a_{2} v_{2}\right)$ follows from $F(0) \leq 0$ and $F^{\prime}(\tau)>0, \forall \tau \geq 0$. The existence of two positive roots to $G\left(\tau_{\phi} ; \rho^{f}\right)=0$ (here the second argument, $\rho^{f}$, is a (fixed) parameter) for $w_{1} /\left(a_{1} v_{1}\right) \geq w_{2} /\left(a_{2} v_{2}\right)$ and $\rho_{L}<\rho^{f}<\rho_{S}^{f}$ follows from $G(0)>0$ for $\rho^{r}>\rho_{L}$ and the fact that (letting $\tilde{\tau}$ denote the unique value of $\tau$ at which the global minimum of the strictly convex function $G(\tau)$ occurs) $G\left(\tilde{\tau} ; \rho^{f}\right)=F(\tilde{\tau})<0$ for $\rho^{s}<\rho_{S}{ }^{f}$. The latter is a consequence of $\partial G / \partial \rho^{f}>0$ and $G\left(\tau_{S} ; \rho_{S}{ }^{\gamma}\right)=F\left(\tau_{S}\right)=0$. It should be noted that the fact that $G^{\prime}(\tilde{\tau}$; $\left.\rho^{f}\right)=0$ allows the parameter $\rho^{f}$ to be eliminated from $G\left(\tilde{\tau} ; \rho^{f}\right)$. It also follows that there is no solution (i.e. value of $\left.\tau_{\phi}\right)$ to $G\left(\tau_{\phi} ; \rho^{f}\right)=0$ for $\rho^{f}>\rho_{S}{ }^{f}$. The proof that $\partial \tau_{S} / \partial J_{3}=-\left(\partial F / \partial J_{3}\right) /\left(\partial F / \partial \tau_{S}\right)>0$ follows from $\partial F / \partial \tau_{S}>0$ and $\partial F / \partial J_{3}<0$ (the latter holding since $\left\{\exp \left(-c_{1} \tau\right)-1+c_{1} \tau\right\}>0$ ).

We will now illustrate the structure of the optimal fire-support policies for the first three problems by considering some numerical examples. The basic parameter set used in the numerical computations is shown in Table 2. Numerical results have not been obtained for Problem 4 when $w_{1} /\left(a_{1} v_{1}\right)>w_{2} /\left(a_{2} v_{2}\right)$ because of the difficulty in solving the associated two-point boundary-value problem. The structure of the optimal policy, however, is similar to that for Problems 2 and 3 , although switching times are, in general, very difficult to determine.

\section{TABLE 2. Basic parameter set for numerical examples.}

\begin{tabular}{|c|c|c|c|}
\hline$i$ & $a_{i}^{*}$ & $c_{i} \dagger$ & \multirow{2}{*}{$T=30$ minutes } \\
\cline { 1 - 3 } & 0.020 & 0.06 & $T=3$ \\
\hline 2 & 0.015 & 0.05 & \\
\hline
\end{tabular}

$* a_{i}$ has units of $\left[X_{i}\right.$ casualties $/\left\{\right.$ (minute) $\times$ (number of $\left.\left.\left.Y_{i}\right)\right\}\right]$.

$\dagger c_{i}$ has units of $\left[Y_{i}\right.$ casualties $/\left\{(\right.$ minute $) \times\left(\right.$ number of $\left.\left.\left.Y_{i}\right)\right\}\right]$.

For Problem 1 it is convenient to introduce the "local" force ratio $[36,39] r_{t}=x_{t} / y_{t}$, which represents the ratio of the numbers of opposing infantry in each of the two combat areas (sec Figure 1). The optimal fire-support policy is most conveniently expressed as an open-loop control in terms of the two initial force ratios, denoted as $r_{i}{ }^{o}=r_{i}(0)$ for $i=1,2$, and the given length of 
time for the approach to contact, $T$. Let us take $\alpha_{1}=\alpha_{2}=1.0$. Then the optimal fire-support policy is graphically depicted in Figure 2. In the initial force-ratio space, the line with equation

$$
r_{2}^{0}=R \gamma \frac{a_{2}}{a_{1}} r_{1}^{0}-\mu a_{2}
$$

where

$$
\begin{aligned}
& R=\alpha_{1} a_{1} c_{1} /\left(\alpha_{2} a_{2} c_{2}\right), \\
& \gamma=\left(\frac{c_{2}}{c_{1}}\right)\left(\frac{e^{c_{1} T}-1}{e^{c_{2} T}-1}\right),
\end{aligned}
$$

and

$$
\mu=\left(\frac{c_{2}}{e^{c_{2} T}-1}\right)\left\{\frac{R}{c_{1}^{2}}\left(e^{c_{1} T}-1-c_{1} T\right)-\frac{1}{c_{2}^{2}}\left(e^{c_{2} T}-1-c_{2} T\right)\right\}
$$

is a "dispersal line" [18, 30, or 40] away from which all optimal battle trajectories flow. This is shown in Figure 3. In other words, the same return is obtained from using $\phi^{*}=0$ or 1 all of the time This dispersal line is determined by equating the extremal returns as a function of initial conditions for these two policies (see Section 4.3). In constructing Figure 3, we have used facts like the following: when $\phi=1$ for $0 \leq t \leq T$ and $r_{2}^{f}=0$, then

$$
r_{1}=\frac{1}{c_{1}}\left\{\left(c_{1} r_{1}^{s}-a_{1}\right) e^{-c_{1} r_{2} / a_{2}}+a_{1}\right\}
$$

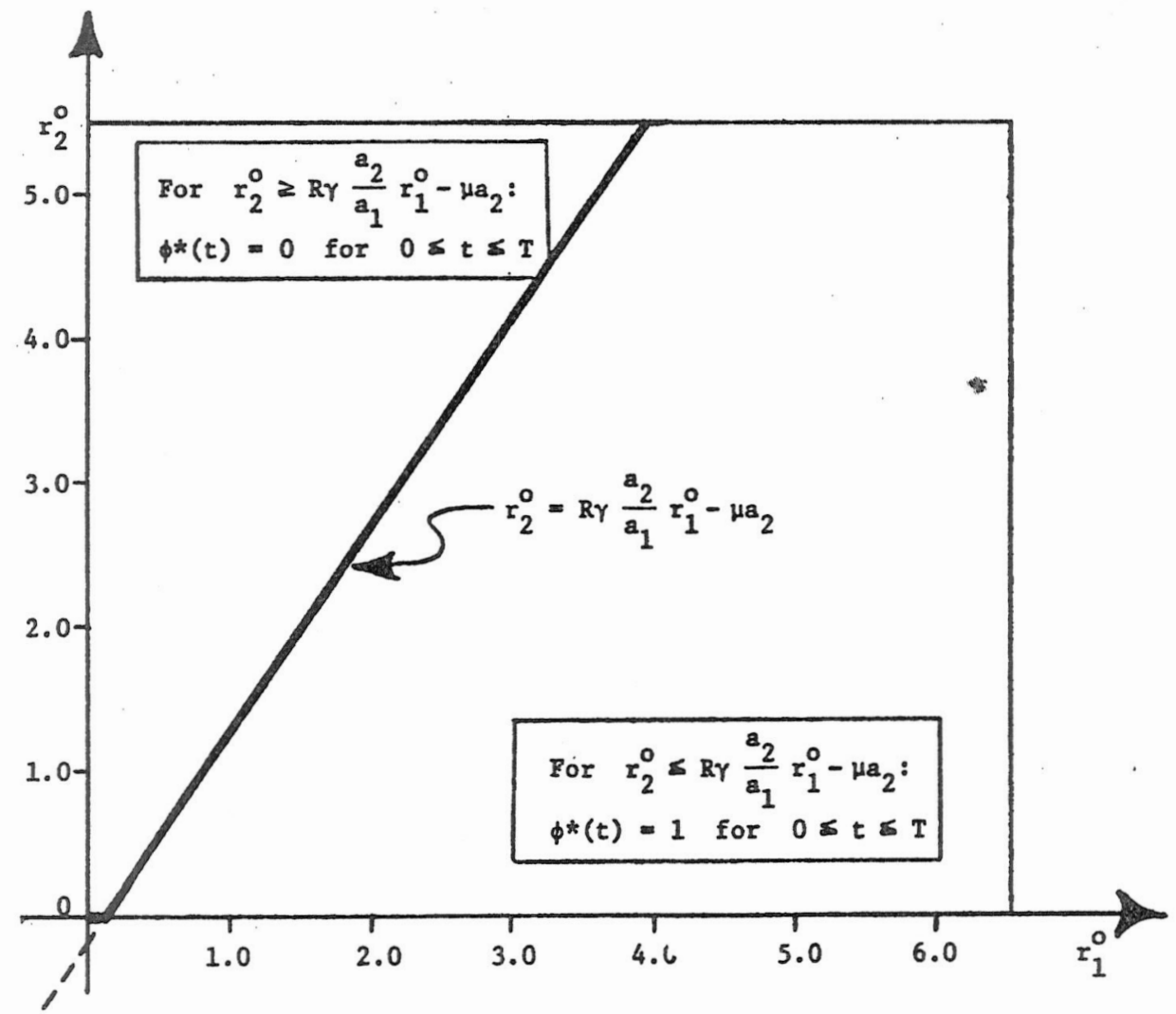

FigURe 2. Optimal (open-loop) fire-support policy for Problem I. 


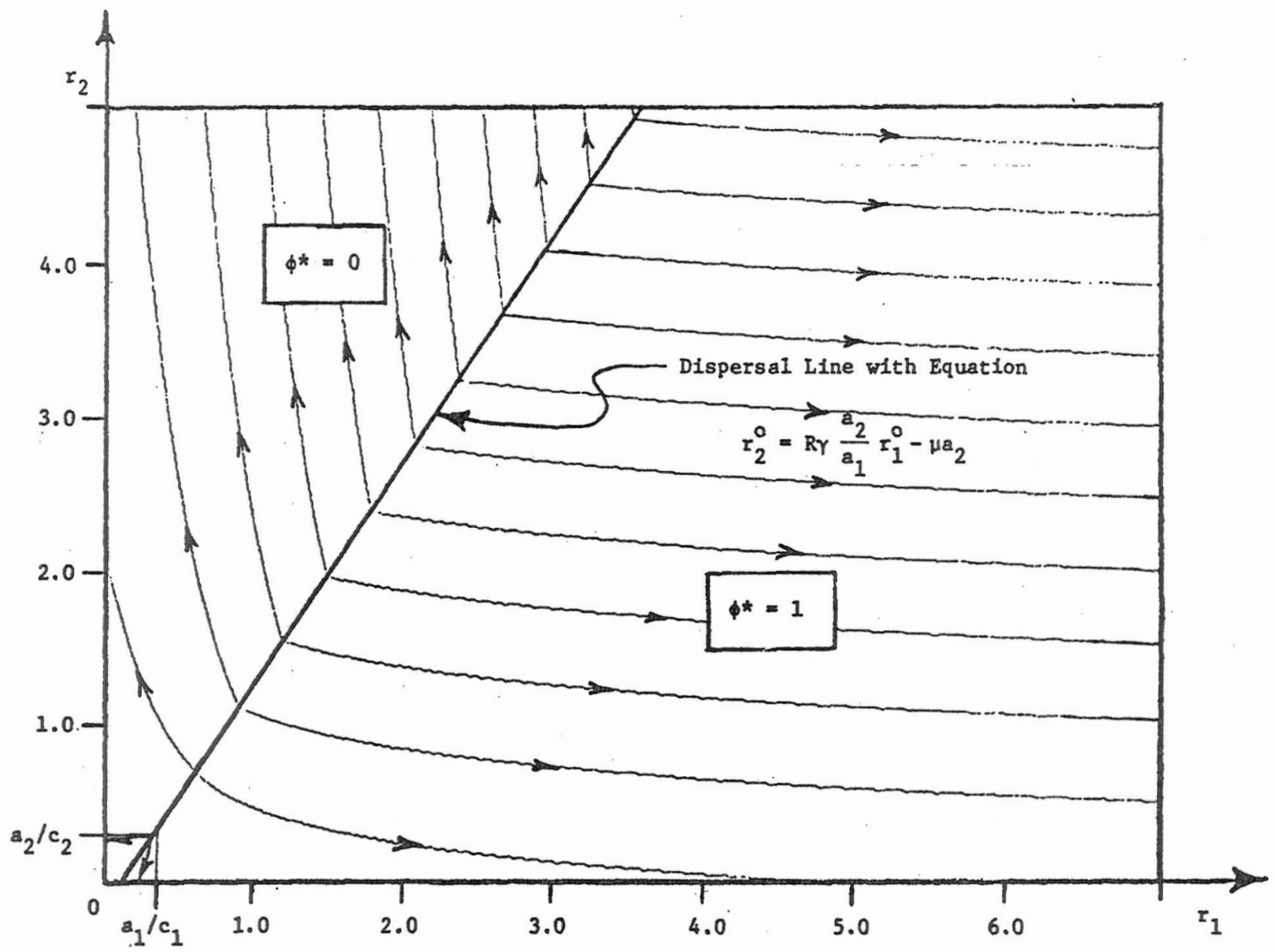

FIGURd 3. Optimal battle trajectories resulting from optimal (open-loop) fire-support policy for Problem 1.

For Problems 2, 3, and 4, the optimal fire-support policy (expressed as a closed-loop control (see [16] or [35])) is most conveniently expressed in terms of $y_{1} / y_{2}$ (i.e. the ratio of the numerical strengths of the two defending infantry forces) and $\tau=T-t$ (i.e. the "balkwards" time or "time to go" in the approach to contact). When enemy forces are valued in direct proportion to the rate at which they destroy value of the friendly forces, i.e.

$$
w_{i}=k a_{i} v_{i} \quad \text { for } i=1,2 \text {, }
$$


the optimal fire-support policy takes a particularly simple form (denoted as policy A):

POLICY A: For $0 \leq t \leq T$

$$
\phi^{*}(t, x, y)= \begin{cases}1 & \text { for } \dot{y}_{1} / y_{2}>a_{2} c_{2} v_{2} /\left(a_{1} c_{1} v_{1}\right) \\ c_{2} /\left(c_{1}+c_{2}\right) & \text { for } y_{1} / y_{2}=a_{2} c_{2} v_{2} /\left(a_{1} c_{1} v_{1}\right) \\ 0 & \text { for } y_{1} / y_{2}<a_{2} c_{2} v_{2} /\left(a_{1} c_{1} v_{1}\right)\end{cases}
$$

This is shown pictorially in Figure 4 in which optimal trajectories are traced backwards in time. It is convenient to note that, for example, when $\phi(\tau)=$ CONSTANT for $0 \leq \tau \leq \sigma$, we have

$$
\rho(\tau)=\rho^{\jmath} \exp \left\{\left[\phi c_{1}-(1-\phi) c_{2}\right] \tau\right\} .
$$

In this case, $\tau_{1}=0$ (see (9), (11), and (15) above), i.e. the entire approach to contact is "Phase I.

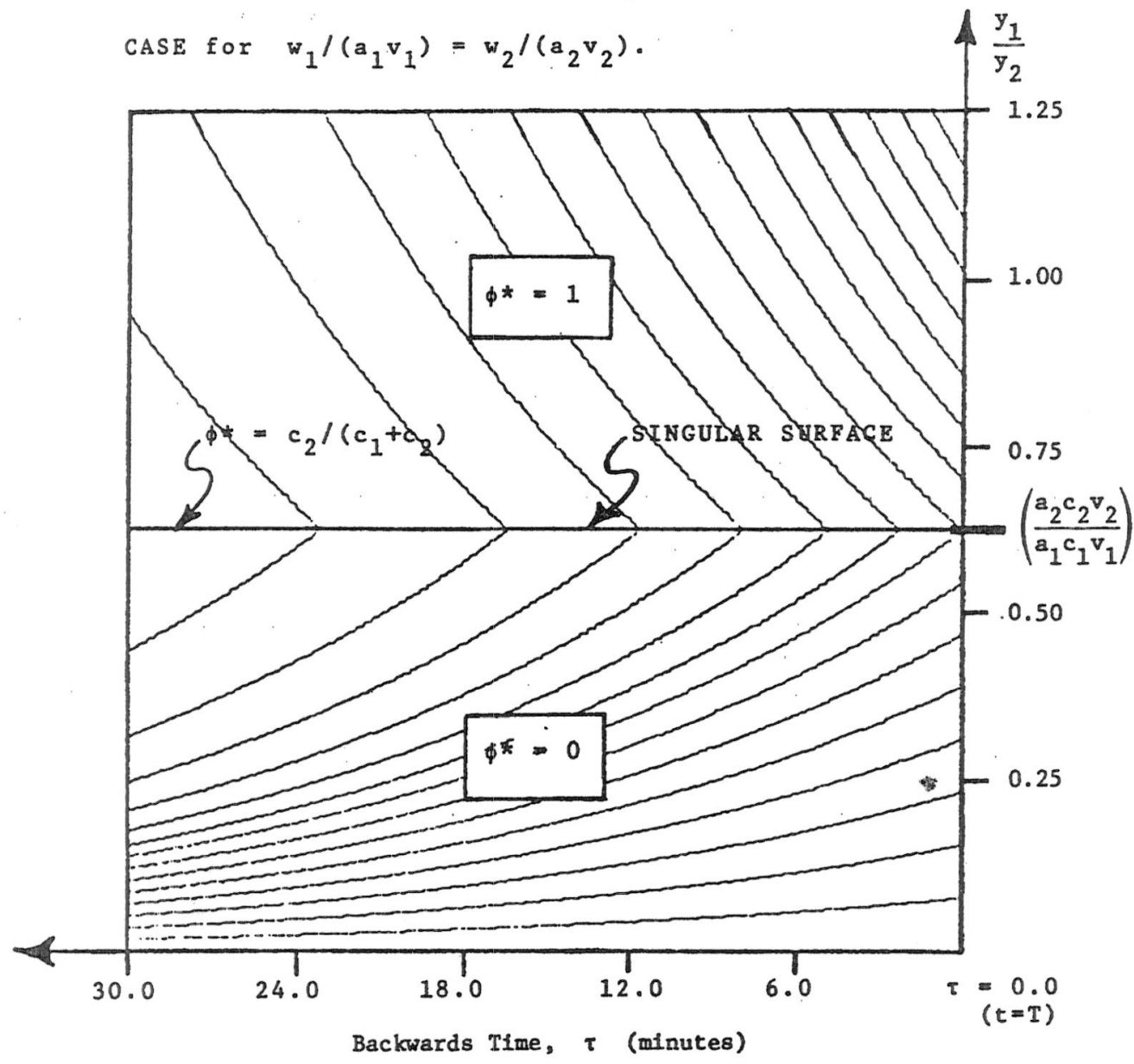

Figure 4. Diagram of optimal (closed-loop) fire-support policy (Policy A) for Problems 2, 3, and 4 when $w_{1} /\left(a_{2} v_{1}\right)$ $=v_{2} /\left(a_{2} v_{2}\right)$.

When enemy forces are not valued in direct proportion to the rate at which they destroy value of the friendly forces (without loss of generality we may assume that $\left.w_{1} /\left(a_{1} v_{1}\right)>w_{2} /\left(a_{2} v_{2}\right)\right)$, the solutions to Problems 2 and 3 are considerably more complex, as shown in Figure 5. For constructing this figure, we have taken $v_{1}=v_{2}=15.0, w_{1}=4.0$, and $w_{2}=1.5$, with other parameter values the same as shown in Table 2. As we have seen above, the planning horizon may be considered to consist of 
two phases (denoted as Phase I and as Phase II), during each of which a different fire-support allocation rule is optimal. We denote this overall optimal policy as Policy B (see (6) and (8)). During Phase I, Policy A is optimal; whereas during Phase II, it is optimal to always concentrate all artillery fire on $Y_{1}$ (which has been valued disproportionately high).

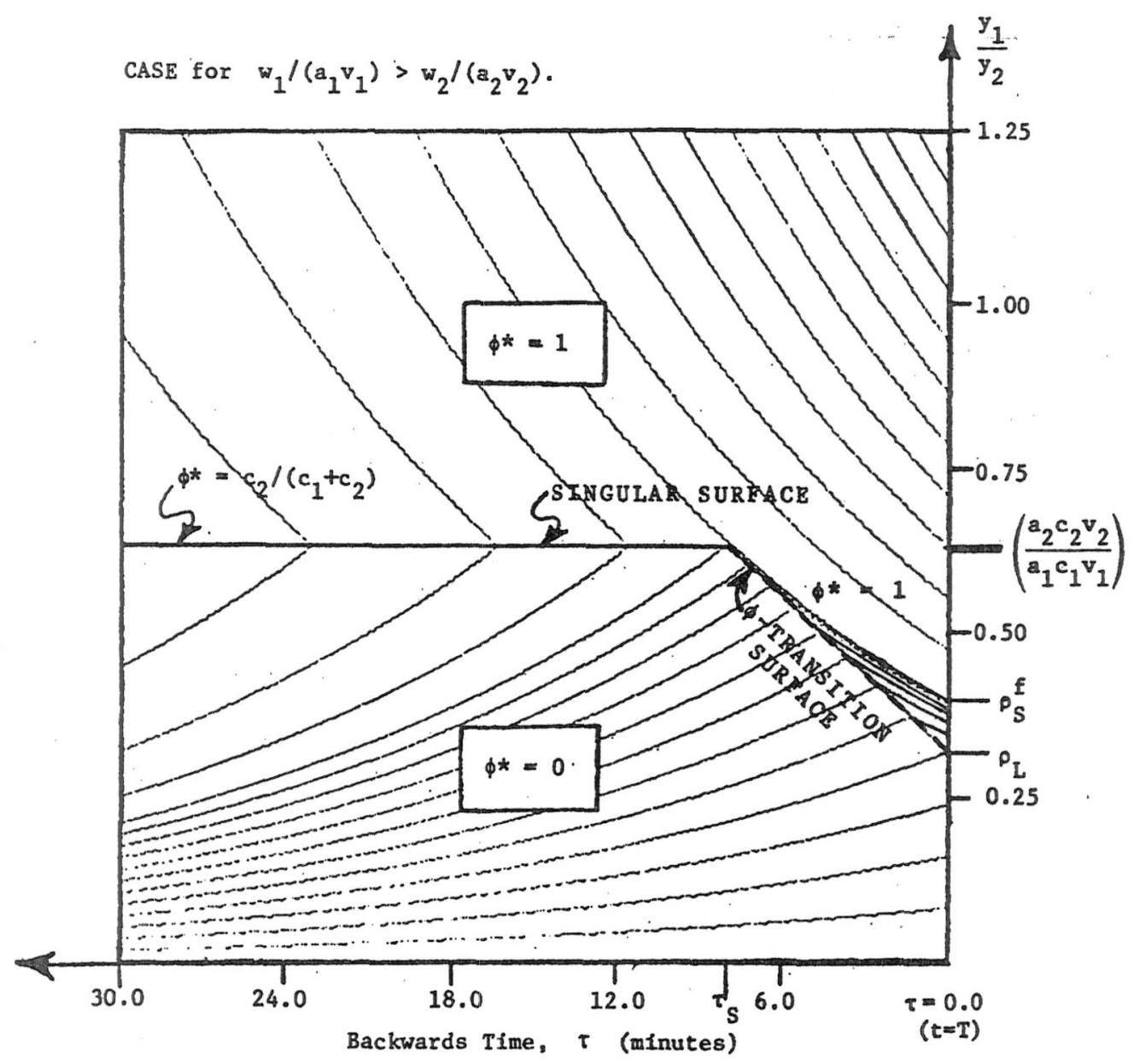

FIGURE 5. Diagram of optimal (closed-loop) fire-support policy (Policy B) for Problem 2 when $w_{1} /\left(a_{1} v_{1}\right)>w_{2} /\left(a_{2} v_{2}\right)$. (The structure of the optimal fire-support policy is similar for Problems 3 and 4.)

The absence or presence of Phase II itself in the optimal time-sequential fire-support policy depends on the ratio of enemy infantry strengths $\rho=y_{1} / y_{2}$. For Problem 2, the length of Phase II (i.e. $\tau_{1}$ ) is independent of the final force levels of the attacking friendly infantry units (i.e. $x_{1}{ }^{f}$ and $x_{2}{ }^{f}$ ) and depends only on $\rho^{f}=y_{1}^{f} / y_{2}^{f}$ and the combat effectiveness parameters (see equations (1)), whereas for Problem 3 the length of Phase II does depend directly on $x_{1}{ }^{f}$ and $x_{2}{ }^{f}$ through the criterion functional $J_{3}=\left\{\Sigma_{k=1}^{2} v_{k} x_{k}^{\gamma}\right\} /\left\{\Sigma_{k=1}^{2} w_{k} y_{k}{ }^{\gamma}\right\}$. Thus, we see that $\tau_{1}$ may be quite different for Problems 2 and 3 : for example, for the parameter set shown in Table 2 (plus force utility values $v_{1}=v_{2}=15.0$, $w_{1}=4.0$, and $w_{2}=1.5$, and terminal values $x_{1}{ }^{\gamma}=x_{2}{ }^{f}=200.0$ and $\left.y_{2}{ }^{\gamma}=50.0\right)$, we have $\tau_{s}($ Problem 2$)=$ 7.93 minutes, while $\tau_{S}$ (Problem 3 ) $=11.37$ minutes. (For computing $\tau_{S}$ (Problem 3) by using $F(\tau)$ given by (15), we have used the fact that

$$
\left(y_{1}^{f}\right)_{S}=y_{2}^{s}\left(\frac{a_{2} c_{2} v_{2}}{a_{1} c_{1} v_{1}}\right) \exp \left(-c_{1} \tau_{S}\right)
$$


to eliminate $y_{1}{ }^{\rho}$ from $J_{3}$ ) Recalling (19) and observing that

$$
\lim _{J_{3} \rightarrow+\infty} \tau_{S}(\operatorname{Problem} 3)=\left(1 / c_{1}\right) \ln \left\{\left[\frac{\left[w_{1} /\left(a_{1} v_{1}\right)\right]}{\left[w_{2} /\left(a_{2} v_{2}\right)\right]}\right\}\right.
$$

we see that for this parameter set the largest that $\tau_{S}$ (Problem 3) may be is $-\lim _{J_{3} \rightarrow+\infty} \tau_{S}$ (Problem 3$)=$ 11.55 minutes. Thus, for this parameter set, $\tau_{S}($ Problem 2$)$ and $\tau_{S}($ Problem 3$)$ may differ by at most fifty percent.

\subsection{Discussion of Comparison}

In this section we will contrast the structure of the optimal fire-support policies for the four problems considered above. Let us recall that in all cases we have assumed that $x_{1}{ }^{f}, x_{2}{ }^{f}>0$.

For Problem 1 the optimal fire-support policy is to always concentrate all artillery fire (i.e. supporting fires) on just one of the two opposing enemy infantry units. This policy will maximize the force ratio at the end of the approach to contact in one of the combat areas (i.e. $x_{i}^{\gamma} / y_{i}^{\prime}$ ) and may be considered to be a "breakthrough" tactic. In other words, one concentrates all fire support on the key enemy unit in order to overwhelm it and effect a penetration.

On the other hand, for Problems 2, 3, and 4 the optimal fire-support policy may involve splitting of fires between the two enemy troop concentrations. This property of the solution has been anticipated in Taylor's earlier work on the optimal control of "linear-law" Lanchester-type attrition processes [31,32] (see also [43]). We may consider this policy to be an "attrition" tactic which aims to wear down the overall enemy strength. The structures of the optimal policies for Problems 2, 3, and 4 are similar, although the switching times (i.e. $\tau_{\phi}$ and $\tau_{S}$ ) may be appreciably different when enemy forces are not valued in direct proportion to the rate at which they destroy value of the friendly forces. In such a case we may assume without loss of generality that inequality holds in (5), i.e.

$$
w_{1} /\left(a_{1} v_{1}\right)>w_{2} /\left(a_{2} v_{2}\right)
$$

The functional dependences of these switching times are also different in Problems 2, 3, and 4. For Problem 2 the switching times (i.e. the $\phi$-transition surface) are independent of the force levels of the attacking friendly forces (i.e. $x_{1}$ and $x_{2}$ ), as is the optimal policy itself. For Problem 3 the switching times depend (see (15) and (16) above) on the ratio of military worths of surviving infantry forces (computed using linear utilities), i.e. $J_{3}=\left\{\Sigma_{k=1}^{2} v_{k} x_{k}(T)\right\} /\left\{\Sigma_{k=1}^{2} w_{k} y_{k}(T)\right\}$. It has been shown (see Section 3.3 above) that $\partial \tau_{s} / \partial J_{3}>0$ so that the larger $J_{3}$ becomes, the more time is spent concentrating fire on $Y_{1}$, although there is an upper limit to this time (see (7)). Similar results hold for Problem 4, only with $J_{3}$ replaced by $\left(-J_{4}\right)$. For comparing the switching times between Problems 3 and 4 , we note that $J_{3}>\left(-J_{4}\right)$ if and only if $J_{3}>\left\{\Sigma_{k=1}^{2} v_{k} x_{k}^{o}\right\} /\left\{\Sigma_{k=1}^{2} w_{k} y_{k}{ }^{o}\right\}$.

The most significant thing to be noted in comparing the optimal fire-support policies* for these four problems is that the entire structure of the optimal policy may be changed merely by changing the criterion functional. In particular, singular subarcs (i.e. the splitting of $W$ 's fire

\footnotetext{
*The referee has insightfully pointed out that it would be interesting to look at the payoff under one criterion corresponding to the optimal control under another criterion. However, this paper emphasizes the structure of the optimal policy rather than the effects on the payoff.
} 
between $Y_{1}$ and $Y_{2}$ ) do not appear in the solution to Problem 1, even though the necessary conditions for optimality on singular subarcs are exactly the same in all four of these problems. Such singular subarcs are, of course, part of the solution for Problems 2, 3, and 4.

\section{DEVELOPMENT OF OPTIMAL POLICY FOR PROBLEM-1}

The optimal policy is developed by application of modern optimal control theory. For Problem 1 it is convenient to introduce the force ratio in the $i$ th combat zone $r_{i}=x_{i} / y_{i}$. Then Problem 1 may be written as

$$
\begin{aligned}
& \underset{\phi_{i}(t)}{\operatorname{maximize}} \sum_{k=1}^{2} \alpha_{k} r_{k}(T) \quad \text { with } T \text { specified, } \\
& \text { subject to: } \frac{d r_{i}}{d t}=-a_{i}+\phi_{i} c_{i} r_{i} \quad \text { for } i=1,2, \\
& \phi_{1}+\phi_{2}=1, \quad \phi_{i} \geq 0, \text { and } r_{i} \geq 0 \quad \text { for } i=1,2,
\end{aligned}
$$

where we recall (2). We also recall that we have assumed that $r_{i}>0$.

\subsection{Necessary Conditions of Optimality}

The Hamiltonian [8] is given by (using (2))

$$
H=\lambda_{1}\left(-a_{1}+\phi c_{1} r_{1}\right)+\lambda_{2}\left(-a_{2}+(1-\phi) c_{2} r_{2}\right),
$$

so that the maximum principle yields the extremal control law

$$
\phi^{*}(t)=\left\{\begin{array}{l}
1 \text { for } S_{\phi}(t)>0 \\
0 \text { for } S_{\phi}(t)<0
\end{array}\right.
$$

where $S_{\phi}(t)$ denotes the $\phi$-switching function defined by

$$
S_{\phi}(t)=c_{1} \lambda_{1} r_{1}-c_{2} \lambda_{2} r_{2}
$$

The adjoint system of equations (again using (2) for convenience) is given by (assuming that $\left.r_{i}(T)>0\right)$

$$
\dot{\lambda}_{i}=-\phi_{i}^{*} c_{i} \lambda_{i} \quad \text { with } \lambda_{i}(T)=\alpha_{i} \quad \text { for } i=1,2 \text {. }
$$

Computing the first two time derivatives of the switching function

$$
\dot{S}_{\phi}(t)=-a_{1} c_{1} \lambda_{1}+a_{2} c_{2} \lambda_{2}, \text { and } \dot{S}_{\phi}(t)=a_{1} c_{1} \lambda_{1}\left(c_{1} \phi\right)-a_{2} c_{2} \lambda_{2}\left(c_{2}(1-\phi)\right)
$$

we see that on a singular subarc (see [32] for a further discussion) we have [8, 21]

$$
r_{1} / a_{1}=r_{2} / a_{2}, \quad \text { and } \quad a_{1} c_{1} \lambda_{1}=a_{2} c_{2} \lambda_{2},
$$

with the singular control given by

$$
\phi_{S}=c_{2} /\left(c_{1}+c_{2}\right)
$$


On such a singular subarc the generalized Legendre-Clebsch condition is satisfied, since

$$
\frac{\partial}{\partial \phi}\left\{\frac{d^{2}}{d t^{2}}\left(\frac{\partial H}{\partial \phi}\right)\right\}=a_{1} c_{1} \lambda_{1}\left(c_{1}+c_{2}\right)>0
$$

\subsection{Synthesis of Extremals}

By an extremal we mean a trajectory on which the necessary conditions of optimality are satisfied. In synthesizing extremals by the usual backwards construction procedure (see, for example, [30] or [32]), it is convenient to introduce the "backwards" time defined by $\tau=T-t$. Rather than explicitly constructing extremals and determining domains of controllability [30, 35,40 , it is more convenient to show that the return (i.e. value of the criterion functional) corresponding to certain extremals dominates that from others. For this purpose it suffices to determine all possible types of extremal policies, as we will now do.

To this end, we write

$$
S_{\phi}(0)=\alpha_{2} a_{2} c_{2}\left(R r_{1}^{\gamma} / a_{1}-r_{2}^{\gamma} / a_{2}\right),
$$

where

$$
R=\alpha_{1} a_{1} c_{1} /\left(\alpha_{2} a_{2} c_{2}\right) \text {. }
$$

Without loss generality we may assume that $R \geq 1$. Then by (31) we have

$$
\stackrel{\circ}{S}_{\phi}(0)=\alpha_{1} a_{1} c_{1}-\alpha_{2} a_{2} c_{2} \geq 0,
$$

where $\stackrel{S}{S}_{\phi}$ denotes the "backwards" time derivative ${\stackrel{\circ}{S_{\phi}}}=d S_{\phi} / d \tau$. Considering (31), we may write

$$
\dot{S}_{\phi}(\tau)=\alpha_{2} a_{2} c_{2}\left\{R\left(\lambda_{1} / \alpha_{1}\right)-\left(\lambda_{2} / \alpha_{2}\right)\right\} \text {. }
$$

It follows that $S_{\phi}(\tau)>0$ and $\phi^{*}(\tau)=1, \forall \tau>0$ when $S_{\phi}(0) \geq 0$ for $R>1$ (also when $S_{\phi}(0)>0$ for $R=1)$. We also have $S_{\phi}(\tau)<0$ and $\phi^{*}(\tau)=0, \forall \tau \geq 0$ when $S_{\phi}(0)<0$ for $R=1$

There may be a change in the sign of $S_{\phi}(\tau)$, however, when $S_{\phi}(0)<0$ for $R>1$. In this case $\phi^{*}(\tau)=0$ for $0 \leq \tau \leq \tau_{1}$ and then

$$
S_{\phi}(\tau)=\alpha_{2} a_{2} c_{2}\left\{R r_{1}(\tau) / a_{1}-\left[\exp \left(c_{2} \tau\right)\right] r_{2}(\tau) / a_{2}\right\}
$$

where $\tau_{1}$ denotes the smallest value of $\tau$ such that $S_{\phi}\left(\tau_{1}\right)=0$. It is clear that we must have $\dot{S}_{\phi}\left(\tau_{1}\right) \geq 0$. If $S_{\phi}\left(\tau_{1}\right)>0$, then we have a transition surface, and from (38) we find that

$$
R r_{1}\left(t_{1}\right) / a_{1}-\left[\exp \left(c_{2} \tau_{1}\right)\right] r_{2}\left(t_{1}\right) / a_{2}=0,
$$

where $t_{1}=T-\tau_{1}$. From (37) we find that

$$
0 \leq \tau_{1}<\left(1 / c_{2}\right) \ln R
$$

If $\dot{S}_{\phi}\left(\tau_{1}\right)=0$, the singular subarc may be entered, and then we have

$$
\tau_{1}=\left(1 / c_{2}\right) \ln R
$$


In this case we have

$$
r_{2}^{f}=R r_{1}^{\jmath} a_{2} / a_{1}+F(R) a_{2} / c_{2}
$$

where $r_{i}{ }^{j}=r_{i}(T)$ and $F(R)=1+R(\ln R-1)$. We easily see that $F(R)>0$ for $R>1$. When $R=1$, we see that once the singular subarc is entered (in fowards time), it is never exited by an extremal trajectory.

For the purposes of determining the optimal policy it suffices to consider the following four extremal policies for $R \geq 1$ :

$$
\begin{array}{lll}
\text { Policy 0: } & \phi^{*}(t)=0 & \text { for } 0 \leq t \leq T, \\
\text { Policy 1: } & \phi^{*}(t)=1 & \text { for } 0 \leq t \leq T, \\
\text { Policy } B-B: & \phi^{*}(t)= \begin{cases}1 & \text { for } 0 \leq t<T-\tau_{1}, \\
0 & \text { for } T-\tau_{1} \leq t \leq T,\end{cases}
\end{array}
$$

where $0 \leq \tau_{1}<\left(1 / c_{2}\right) \ln R$, and

$$
\text { Policy } S: \quad \phi^{*}(t)= \begin{cases}c_{2} /\left(c_{1}+c_{2}\right) & \text { for } 0 \leq t<T-\tau_{1}, \\ 0 & \text { for } T-\tau_{1} \leq t \leq T,\end{cases}
$$

where $\tau_{1}=\left(1 / c_{2}\right) \ln R$ and $r_{1}{ }^{\circ} / a_{1}=r_{2}{ }^{\circ} / a_{2}$. The only extremal policies that are omitted here are those corresponding to extremals which contain a singular subarc but $r_{1}{ }^{\circ} / a_{1} \neq r_{2}{ }^{\circ} / a_{2}$. It is readily seen from (34) that Policy 0 yields $R r_{1}{ }^{\gamma} / a_{1} \geq r_{2}{ }^{s} / a_{2}$, etc. We also note that corresponding to the bang-bang policy (45) we have

$$
\begin{aligned}
& r_{1}\left(t_{1}\right)=\left\{\left(c_{1} r_{1}^{\circ}-a_{1}\right) \exp \left(c_{1} t_{1}\right)+a_{1}\right\} / c_{1}, \\
& r_{2}\left(t_{1}\right)=r_{2}^{0}-a_{2} t_{1} \geq 0 .
\end{aligned}
$$

\subsection{Determination of the Optimal Fire-Support Policy}

It has not been possible to establish the optimality of a policy by citing one of the many sets of sufficient conditions that are available $[8,32,35]$. In particular, although the planning horizon for the problem at hand is of fixed length, one cannot invoke the sufficient conditions based on convexity of Mangasarian [24] or Funk and Gilbert [11] because the right-hand sides of the differential equations (9) are not concave functions of $r_{i}$ and $\phi_{i}$. As we have discussed elsewhere [31-33, $35,40]$, however, the optimality of an extremal trajectory may be proven via citing the appropriate existence theorem for an optimal control; for the problem at hand there are two further subcases: (1) if the extremal is unique, then it is optimal, or (2) if the extremal is not unique and only a finite number exist, then the optimal trajectory is determined by considering the finite number of corresponding values of the criterion functional. The existence of a measurable optimal control follows by Corollary 2 on p. 262 of [23]. In Sections 4.1 and 4.2 above, we have considered necessary conditions of optimality for piecewise continuous controls (see p. 10 and pp. 20-21 of [28]). It remains to show that the measurable optimal control may be taken to be piecewise continuous. This assertion may be proved by observing that if we consider the maximum principle for measurable controls (see p. 81 of [28]) in the backwards synthesis of extremals, then the optimal control may be taken to be piecewise constant (and hence piecewise continuous). This last assertion follows from the control variable appearing linearly in the Hamiltonian (27), the control variable space being 
compact, and the switching function (29) being continuous for $0 \leq t \leq T$. The maximum principle (also singular control considerations) then yields that the optimal control must be piecewise constant almost everywhere, since $S_{\phi}(t)$ can change sign at most once. Hence, it may be considered to be piecewise constant (see p. 130 of [28]). (The authors wish to thank J. Wingate of the Naval Surface Weapons Center, White Oak, for generously pointing out this type of argument.)

We will now show that the optimal control must be constant. By the principle of optimality [8] it suffices for the purpose of showing that a singular solution is always nonoptimal to consider a singular extremal which begins with a singular subarc. We will show that the returns from both Policy $B-B$ and also Policy $S$ for a given point in the initial state space are dominated by the return corresponding to a constant extremal control. We denote the value of the criterion functional corresponding to Policy 0 as $J_{0}$, that corresponding to Policy $B-B$ as $J_{B}$, etc. Then we have

$$
\begin{aligned}
& J_{0}=\alpha_{2} a_{2} c_{2}\left\{\left(\frac{r_{1}^{0}}{a_{1}}\right) \frac{R}{c_{1}}-\left(\frac{r_{2}^{0}}{a_{2}}\right) \frac{1}{c_{2}} \exp \left(c_{2} T\right)-\left[\frac{R T}{c_{1}}+\frac{1}{c_{2}^{2}}\left(\exp \left(c_{2} T\right)-1\right)\right]\right\}, \\
& J_{1}=\alpha_{2} a_{2} c_{2}\left\{\left(\frac{r_{1}{ }^{0}}{a_{1}}\right) \frac{R}{c_{1}} \exp \left(c_{1} T\right)+\left(\frac{r_{2}^{0}}{a_{2}}\right) \frac{1}{c_{2}}-\left[\frac{R}{c_{1}^{2}}\left(\exp \left(c_{1} T\right)-1\right)+\frac{T}{c_{2}}\right]\right\} \text {, } \\
& J_{B}=\alpha_{2} a_{2} c_{2}\left\{\left(\frac{r_{1}^{0}}{a_{1}}\right) \frac{R}{c_{1}} \exp \left(c_{1}\left[T-\tau_{1}\right]\right)+\left(\frac{r_{2}{ }^{\circ}}{a_{2}}\right) \frac{1}{c_{2}} \exp \left(c_{2} \tau_{1}\right)\right. \\
& \left.-\frac{R}{c_{1}^{2}}\left[\exp \left(c_{1}\left[T-\tau_{1}\right]\right)-1+c_{1} \tau_{1}\right]-\frac{1}{c_{2}^{2}}\left[\left(1+c_{2}\left[T-\tau_{1}\right]\right) \exp \left(c_{2} \tau_{1}\right)-1\right]\right\},
\end{aligned}
$$

and

$$
J_{S}=\alpha_{2} a_{2} c_{2}\left\{\left(\frac{r_{1}{ }^{0}}{a_{1}}\right) \frac{R^{\alpha}}{K} \exp (K T)-\left[\frac{R}{\bar{K}^{2}}\left(R^{-\beta} \exp (K T)-1\right)+\frac{1}{c_{1} c_{2}} R \ln R+\frac{1}{c_{2}{ }^{2}}(R-1)\right]\right\}
$$

where $\alpha=c_{2} /\left(c_{1}+c_{2}\right), \alpha+\beta=1$, and $K=c_{1} c_{2} /\left(c_{1}+c_{2}\right)$. It is convenient to define $\Delta J_{1-0}=J_{1}-J_{0}$, etc., and then

$$
\begin{aligned}
\Delta J_{1-0}=\alpha_{2} a_{2} c_{2}\left\{R \left[\left(\frac{r_{1}{ }^{\circ}}{a_{1}}\right)\left(\frac{\exp \left(c_{1} T\right)-1}{c_{1}}\right)\right.\right. & \left.-\frac{1}{c_{1}^{2}}\left(\exp \left(c_{1} T\right)-1-c_{1} T\right)\right] \\
& \left.-\left[\left(\frac{r_{2}{ }^{0}}{a_{2}}\right)\left(\frac{\exp \left(c_{2} T\right)-1}{c_{2}}\right)-\frac{1}{c_{2}^{2}}\left(\exp \left(c_{2} T\right)-1-c_{2} T\right)\right]\right\},
\end{aligned}
$$

$$
\begin{aligned}
\Delta J_{1-B}=\alpha_{2} a_{2} c_{2}\{R & {\left[\left(\frac{r_{1}^{0}}{a_{1}}\right)\left(\frac{\exp \left(c_{1} T\right)-\exp \left(c_{1}\left[T-\tau_{1}\right]\right)}{c_{1}}\right)-\frac{1}{c_{1}^{2}}\left(\exp \left(c_{1} T\right)-\exp \left(c_{1}\left[T-\tau_{1}\right]\right)-c_{1} \tau_{1}\right)\right] } \\
- & {\left.\left[\left(\frac{r_{2}^{0}}{a_{2}}\right)\left(\frac{\exp \left(c_{2} \tau_{1}\right)-1}{c_{2}}\right)-\frac{1}{c_{2}^{2}}\left(\exp \left(c_{2} \tau_{1}\right)+c_{2}\left[T-\tau_{1}\right] \exp \left(c_{2} \tau_{1}\right)-1-c_{2} T\right)\right]\right\}, }
\end{aligned}
$$

and

$$
\begin{aligned}
\Delta J_{1-S}= & \alpha_{2} a_{2} c_{2}\left\{\left(\frac{r_{1}^{o}}{a_{1}}\right)\left[\frac{1}{c_{1}}\left(R \exp \left(c_{1} T\right)-1\right)-\frac{1}{K}\left(R^{\alpha} \exp (K T)-1\right)\right]\right. \\
& \left.+\frac{R}{K^{2}}\left(R^{-\beta} \exp (K T)-1-\frac{K T}{R}\right)-\frac{R}{c_{1}{ }^{2}}\left(\exp \left(c_{1} T\right)-1-\frac{c_{1} T}{R}\right)+\frac{1}{c_{1} c_{2}} R \ln R+\frac{1}{c_{2}{ }^{2}}(R-1)\right\} .
\end{aligned}
$$

In computing $\Delta J_{1-s}$ we assume that $r_{1}{ }^{\circ} / a_{1}=r_{2}{ }^{\circ} / a_{2}$.

We now state and prove Lemma 1.

LEMMA 1: Assume that $R \geq 1$ and $T \geq \tau_{1}$. If $\Delta J_{1-0} \geq 0$, then $\Delta J_{1-B} \geq 0$. 
PROOF: (a) We consider for $t \geq \tau_{1}$

$$
\begin{aligned}
F^{\prime}(t)=R\left\{\left(\frac{r_{1}{ }^{0}}{a_{1}}\right)\left(\frac{\exp \left(c_{1} t\right)-\exp \left(c_{1}\left[t-\tau_{1}\right]\right)}{c_{1}}\right)-\frac{1}{c_{1}{ }^{2}}\left(\exp \left(c_{1} t\right)-\exp \left(c_{1}\left[t-\tau_{1}\right]\right)-c_{1} \tau_{1}\right)\right\} \\
-\left\{\left(\frac{r_{2}{ }^{0}}{a_{2}}\right)\left(\frac{\exp \left(c_{2} \tau_{1}\right)-1}{c_{2}}\right)-\frac{1}{c_{2}{ }^{2}}\left(\exp \left(c_{2} \tau_{1}\right)+c_{2}\left[t-\tau_{1}\right] \exp \left(c_{2} \tau_{1}\right)-1-c_{2} t\right)\right\} .
\end{aligned}
$$

Then $\Delta J_{1-0} \geq 0 \Leftrightarrow F\left(\tau_{1}\right) \geq 0$.

(b) We compute that

$$
F^{\prime}(t)=R\left\{\exp \left(c_{1} t\right)-\exp \left(c_{1}\left[t-\tau_{1}\right]\right)\right\}\left\{\left(\frac{r_{1}^{o}}{a_{1}}\right)-\frac{1}{c_{1}}\left(1-\exp \left(-c_{1} \tau_{1}\right)\right)\right\}+\frac{1}{c_{2}}\left(\exp \left(c_{2} \tau_{1}\right)-1\right)
$$

(c) If $c_{1} r_{1}^{0} \leq a_{1}$, then $d r_{1} / d t(t) \leq 0$ for $0 \leq t \leq t_{1}$ so that $\left(r_{1}^{o} / a_{1}\right) \geq\left(r_{1}\left(t_{1}\right) / a_{1}\right) \geq \tau_{1}$. It follows that $F^{\prime}(t) \geq 0$. If $c_{1} r_{1}^{o}>a_{1}$, then $F^{\prime}(t)>0$. Thus, we already have $F^{\prime}(t) \geq 0$ for $t \geq \tau_{1}$.

(d) By (a) and (c), we have $F(t) \geq 0$, whence follows the lemma.

Q.E.D.

LEMMA 2: Assume that $R \geq 1$. Then for $t_{1}=T-\tau_{1} \geq 0$, we have $\Delta J_{0-B} \geq 0$ with $\Delta J_{0-B}>0$ for $t_{1}>0$.

PROOF: (a) We consider for $t_{1} \geq 0$

$$
\begin{aligned}
F\left(t_{1}\right)=-R\left\{\left(\frac{r_{1}{ }^{\circ}}{a_{1}}\right)\left(\frac{\exp \left(c_{2} t_{1}\right)-1}{c_{2}}\right)-\right. & \left.\frac{1}{c_{1}^{2}}\left(\exp \left(c_{1} t_{1}\right)-1-c_{1} t_{1}\right)\right\} \\
& +\exp \left(c_{2} \tau_{1}\right)\left\{\left(\frac{r_{2}^{\circ}}{a_{2}}\right)\left(\frac{\exp \left(c_{2} t_{1}\right)-1}{c_{2}}\right)-\frac{1}{c_{2}^{2}}\left(\exp \left(c_{2} t_{1}\right)-1-c_{2} t_{1}\right)\right\} .
\end{aligned}
$$

We observe that $F^{\prime}(0)=0$.

(b) We compute that

$$
F^{\prime}\left(t_{1}\right)=-\frac{R}{a_{1}}\left\{\frac{1}{c_{1}}\left[\left(c_{1} r_{1}{ }^{\circ}-a_{1}\right) \exp \left(c_{1} t_{1}\right)+a_{1}\right]\right\}+\frac{\exp \left(c_{2} \tau_{1}\right)}{a_{2}}\left\{r_{2}^{0} \exp \left(c_{2} t_{1}\right)-\frac{a_{2}}{c_{2}}\left(\exp \left(c_{2} t_{1}\right)-1\right)\right\} .
$$

Considering (22) and (30), we find that for $t_{1} \geq 0$ we have

$$
F^{\prime}\left(t_{1}\right)=\exp \left(c_{2} \tau_{1}\right)\left\{\left(\frac{r_{2}^{0}}{a_{2}}\right)\left(\exp \left(c_{2} t_{1}\right)-1\right)-\frac{1}{c_{2}}\left(\exp \left(c_{2} t_{1}\right)-1-c_{2} t_{1}\right)\right\}
$$

(c) Recalling (47) that $r_{2}^{\circ} / a_{2} \geq t_{1}$, we have for $t_{1} \geq 0$

$$
F^{\prime}\left(t_{1}\right) \geq \exp \left(c_{2} \tau_{1}\right)\left\{t_{1}\left(\exp \left(c_{2} t_{1}\right)-1\right)-\frac{1}{c_{2}}\left(\exp \left(c_{2} t_{1}\right)-1-c_{2} t_{1}\right)\right\} \geq 0,
$$

since for $t \geq 0$ we have $g(t) \geq 0$, where $g(t)=t\left(\exp \left(c_{2} t\right)-1\right)-\left(\exp \left(c_{2} t\right)-1-c_{2} t\right) / c_{2}$. The latter result follows from $g(0)=0$ and $g^{\prime}(t) \geq 0, \forall t \geq 0$.

(d) Thus, $F\left(\dot{t}_{1}\right) \geq 0, \forall t_{1} \geq 0$, whence follows the lemma.

As an immediate consequence of Lemmas 1 and 2 and analogous results for $R \leq 1$, we have Theorem 1.

THEOREM 1: For $T \geq \tau_{1}>0$, we have $\max \left(J_{0}, J_{1}\right) \geq J_{B}$ with strict inequality holding for $T>\tau_{1}$.

We next consider Lemma 3.

IEMMA 3: Assume that $R \geq 1$ and $T \geq \tau_{1}$. Then we have $\Delta J_{1-s} \geq 0$ with $\Delta J_{1-s}>0$ for $R>1$ or $T>\tau_{1}$, 
PROOF: (a) We consider for $t \geq 0$

$$
\begin{array}{r}
F(t)=t\left\{\left(R \exp \left(c_{1} t\right)-1\right) / c_{1}-\left(R^{\alpha} \exp K t-1\right) / K\right\}+R\left(R^{-\beta} \exp (K T)-1-K t / R\right) / K^{2} \\
-R\left(\exp \left(c_{1} t\right)-1-c_{1} T / R\right) / c_{1}{ }^{2}+(R \ln R) /\left(c_{1} c_{1}\right)+(R-1) / c_{2}{ }^{2} .
\end{array}
$$

Then we have

$$
F(0)=R(R-\beta-1) / K^{2}+(R \ln R) /\left(c_{1} c_{2}\right)+(R-1) / c_{2}{ }^{2}=f(R) \geq 0,
$$

with $f(R)>0$ for $R>1$. The latter result follows from $f(1)=f^{\prime}(1)=0$ and $f^{\prime \prime}(R)=\left(1-R^{-\beta}\right) /\left(c_{1} c_{2} R\right)$ $>0, \forall R>1$.

(b) Computing $F^{\prime}(t)=R^{\alpha} t\left\{R^{\beta} \exp \left(c_{1} t\right)-\exp (K t)\right\} \geq R^{\alpha} t\left\{\exp \left(c_{1} t\right)-\exp (K t)\right\}>0$ for $R \geq 1$ and $t>0$, we see from (a) that $F(t ; R) \geq 0$ with $F(t ; R)>0$ for $R>1$ or $t>0$.

(c) We now consider $G(t)=\left(R \exp \left(c_{1} t\right)-1\right\} / c_{1}-\left(R^{\alpha} \exp (K t)-1\right\} / K$. It follows that $G(0)$ $=1 / c_{2}+R / c_{1}-R^{\alpha} / K=g(R) \geq 0$, since $g(1)=0$ and $g^{\prime}(R)=\left(1-R^{-\beta}\right) / c_{1}$. Also, $G^{\prime}(t)=R^{\alpha}\left\{R^{\beta} \exp \left(c_{1} t\right)\right.$ $-\exp (K t)\} \geq 0$. Hence, $G(t) \geq 0$.

(d) Recalling that $r_{1} 0 / a_{1} \geq T$, we have by (c) that $\Delta J_{1-s} \geq \alpha_{2} a_{2} c_{2} F(T ; R) \geq 0$ with $F(T ; R)>0$ for $R>1$ or $T>\tau_{1}$.

From Lemma 3 and the analogous result for $R \leq 1$, Theorem 2 follows.

THEOREM 2: Assume that $T \geq \tau_{1}$. Then $\max \left(J_{0}, J_{1}\right) \geq J_{S}$ with inequality holding for $T>\tau_{1}$.

Thus, we see from Theorems 1 and 2 that the optimal control must be constant and equal to either 0 or 1 for $0 \leq t \leq T$. The results given in Section 3.3 (see, in particular, Figures 2 and 3 ) then follow from consideration of $\Delta J_{1-0}$ (see (52)).

\section{DEVELOPMENT OF OPTIMAL POLICY FOR PROBLEM 2}

In this case we consider (1) with the criterion function

$$
J_{2}=\sum_{k=1}^{2} v_{k} x_{k}(T)-\sum_{k=1}^{2} w_{k} y_{k}(T)
$$

Thus, for this problem the state space (considering time to be an additional state variable) is fivedimensional.

\subsection{Necessary Conditions of Optimality}

The Hamiltonian [8] is given by (using (2))

$$
H=-\sum_{i=1}^{2} p_{i} a_{i} y_{i}-q_{1} \phi c_{1} y_{1}-q_{2}(1-\phi) c_{2} y_{2},
$$

so that the maximum principle yields the extremal control law

$$
\phi^{*}(t)= \begin{cases}1 & \text { for } S_{\phi}(t)>0 \\ 0 & \text { for } S_{\phi}(t)<0\end{cases}
$$


where $S \phi(t)$ denotes the $\phi$-switching function defined by

$$
S_{\phi}(t)=c_{1}\left(-q_{1}\right) y_{1}-c_{2}\left(-q_{2}\right) y_{2}
$$

The adjoint system of equations (again using (2) for convenience) is given by (assuming that $\left.x_{i}(T)>0\right)$

$$
\begin{gathered}
p_{i}(t)=v_{i} \text { for } 0 \leq t \leq T \text { with } i=1,2, \text { and } \\
\dot{q}_{i}=a_{i} v_{i}+\phi_{i}^{*} c_{i} q_{i} \quad \text { with } q_{i}(T)=-w_{i} \text { for } i=1,2 .
\end{gathered}
$$

Computing the first two time derivatives of the switching function

$$
\dot{S}_{\phi}(t)=-a_{1} c_{1} v_{1} y_{1}+a_{2} c_{2} v_{2} y_{2}, \quad \text { and } \ddot{S}_{\phi}(t)=a_{1} c_{1} v_{1} y_{1}\left(c_{1} \phi\right)-a_{2} c_{2} v_{2} y_{2}\left(c_{2}(1-\phi)\right),
$$

we see that on a singular subarc we have $[8,21]$

$$
y_{1} / y_{2}=a_{2} c_{2} v_{2} /\left(a_{1} c_{1} v_{1}\right), \text { and }\left(-q_{1}\right) /\left(a_{1} v_{1}\right)=\left(-q_{2}\right) /\left(a_{2} v_{2}\right),
$$

with the singular control given by.

$$
\phi_{S}=c_{2} /\left(c_{1}+c_{2}\right) .
$$

On such a singular subarc the generalized Legendre-Clebsch condition is satisfied, since

$$
\frac{\partial}{\partial \phi}\left\{\frac{d^{2}}{d t^{2}}\left(\frac{\partial H}{\partial \phi}\right)\right\}=a_{1} c_{1} v_{1} y_{1}\left(c_{1}+c_{2}\right)>0
$$

For Problem 1 it was convenient to consider a "reduced" state space consisting of $t, r_{1}=x_{1} / y_{1}$, and $r_{2}$, while for Problem 2 we are considering the "full" state space of $t, x_{1}, x_{2}, y_{1}$, and $y_{2}$. It seems appropriate to point out the corresponding relation between the adjoint variables in these two state spaces. This relation is easily seen by considering the optimal return function [8], denoted as $W$, and the following transformation of variables:

$$
t=t \text { and } r_{i}=x_{i} / y_{i} \text { for } i=1,2 \text {. }
$$

Then we have, for example,

so that we obtain

$$
p_{i}(t)=\frac{\partial W}{\partial x_{i}(t)}=\frac{\partial W}{\partial r_{i}} \frac{\partial r_{i}}{\partial x_{i}}
$$

$$
p_{i}=\lambda_{i} / y_{i} \text { and } q_{i}=-r_{i} \lambda_{i} / y_{i} \text { for } i=1,2 \text {. }
$$

Let us also note that, alternatively, Problem 1 could have been solved in the "full" state space of $t, x_{1}, x_{2}, y_{1}$, and $y_{2}$, while Problem 2 cannot be solved in the "reduced" state space. The latter conclusion follows from considering (58) and the requirement (see (63) above) that $p_{i} / q_{i}=-1 / r_{i}$ must hold for the transformation (62) to be applicable.

\subsection{Synthesis of Extremals}

In synthesizing extremals by the usual backwards construction procedure it is convenient to consider 


$$
S_{\phi}(0)=a_{2} c_{2} v_{2} y_{2}^{f}\left(\frac{w_{1}}{a_{1} v_{1}}\right)\left\{\frac{a_{1} c_{1} v_{1} y_{1}^{f}}{a_{2} c_{2} v_{2} y_{2}^{f}}-\left(\frac{w_{2}}{a_{2} v_{2}}\right) /\left(\frac{w_{1}}{a_{1} v_{1}}\right)\right\}
$$

and

$$
\stackrel{\circ}{S}_{\phi}(\tau)=a_{1} c_{1} v_{1} y_{1}-a_{2} c_{2} v_{2} y_{2}
$$

and

where $\tau$ denotes the "backwards" time defined by $\tau=T-t$, and $\stackrel{S}{\phi}_{\phi}$ denotes the "backwards"

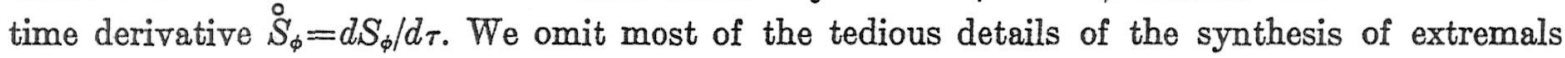
because of similarity to those in [32]. Without loss of generality we may assume that (5) holds, and then there are two cases to be considered: (I) $w_{1} /\left(a_{1} v_{1}\right)=w_{2} /\left(a_{2} v_{2}\right)$, and (II) $w_{1} /\left(a_{1} v_{1}\right)>w_{2} /\left(a_{2} v_{2}\right)$.

CASE I: $w_{1} /\left(a_{1} v_{1}\right)=w_{2} /\left(a_{2} v_{2}\right)$; i.e. $w_{i}=k a_{i} v_{i}$ for $i=1,2$.

In this case (64) becomes

$$
S_{\phi}(0)=a_{2} c_{2} v_{2} y_{2}^{f}\left(w_{1} /\left(a_{1} v_{1}\right)\right)\left\{a_{1} c_{1} v_{1} y_{1}^{f} /\left(a_{2} c_{2} v_{2} y_{2}^{\jmath}\right)-1\right\},
$$

whence follows the synthesis of extremals shown in Figure 4.

CASE II: $w_{1} /\left(a_{1} v_{1}\right)>w_{2} /\left(a_{2} v_{2}\right)$.

In this case it follows from (56), (64), and (65) that for $\rho^{\rho}=y_{1}{ }^{\gamma} / y_{2}{ }^{p} \geq a_{2} c_{2} v_{2} /\left(a_{1} c_{1} v_{1}\right)$, we have $S_{\phi}(\tau)>0$ and $\phi^{*}(\tau)=1$ for all $\tau>0$. Since $S_{\phi}(0) \leq 0 \Rightarrow{\stackrel{S}{S_{\phi}}}_{(0)}<0$, it follows that for

$$
\rho^{f} \leq\left(\frac{a_{2} c_{2} v_{2}}{a_{1} c_{1} v_{1}}\right)\left(\frac{w_{2}}{a_{2} v_{2}}\right) /\left(\frac{w_{1}}{a_{1} v_{1}}\right)
$$

we have $S_{\phi}(\tau)<0$ and $\phi^{*}(\tau)=0$ for all $\tau>0$.

There may be a change in the sign of $S_{\phi}(\tau)$, however, for $c_{2} w_{2} /\left(c_{1} w_{1}\right)<\rho^{f}<a_{2} c_{2} v_{2} /\left(a_{1} c_{1} v_{1}\right)$. In this case $\phi^{*}(\tau)=1$ for $0 \leq \tau \leq \tau_{1}$ and then

$$
S_{\phi}(\tau)=a_{2} c_{2} v_{2} y_{2}^{\rho}\left\{\frac{1}{c_{1}}\left[\exp \left(c_{1} \tau\right)-1\right]\left(\frac{a_{1} c_{1} v_{1}}{a_{2} c_{2} v_{2}}\right) \rho^{f}-\tau+\left(\frac{a_{1} c_{1} v_{1}}{a_{2} c_{2} v_{2}}\right)\left(\frac{w_{1}}{a_{1} v_{1}}\right) \rho^{\rho}-\left(\frac{w_{2}}{a_{2} v_{2}}\right)\right\}
$$

It is clear that we must have $S_{\phi}\left(\tau_{1}\right) \leq 0$. If $S_{\phi}\left(\tau_{1}\right)<0$, then we have a tansition surface with $\tau_{1}$ (denoted as $\tau_{\phi}$ ) given by the smaller of the two positive roots of $G\left(\tau_{\phi} ; \rho^{f}\right)=0$, where $G\left(\tau ; \rho^{f}\right)$ is given by (12). If $\dot{S}_{\phi}\left(\tau_{1}\right)=0$, a singular subarc may be entered, and then we have that $\tau_{1}$ (denoted as $\tau_{S}$ ) is given by the unique nonnegative root of $F\left(\tau_{S}\right)=0$, where $F(\tau)$ is given by (11). We denote the corresponding value of $\rho^{f}$ as $\rho_{S}{ }^{f}$. Then there is no switch in $\phi^{*}$ for $\rho^{f}>\rho_{S}{ }^{f}$. We state this result as Theorem 3.

THEOREM $3: \phi^{*}(\tau)=1$ for all $\tau \geq 0$ when $\rho^{\gamma}>\rho_{s}{ }^{\gamma}$.

PROOF: Immediate by $G\left(\tau_{S} ; \rho_{S}\right)=F\left(\tau_{S}\right)=0$ and $\partial G / \partial \rho^{f}>0$, since then there is no solution to $G\left(\tau_{1} ; \rho^{t}\right)=0$ for $\rho^{r}>\rho_{S}{ }^{j}$.

Q.E.D.

The bounds on $\tau_{S}$ given by (13) and (14) are developed as follows. First assume that $w_{1} /\left(a_{1} v_{1}\right) \leq$ $1 / c_{1}$ and consider $F(\tau)=\tau+\left\{1 / c_{1}-w_{1} /\left(a_{1} v_{1}\right)\right\} \exp \left(-c_{1} \tau\right)-\left\{1 / c_{1}-w_{2} /\left(a_{2} v_{2}\right)\right\}$. Then $c_{1} w_{1} /\left(a_{1} v_{1}\right) \leq F^{\prime}(\tau)$ $\leq 1$ and $F^{\prime \prime}(\tau) \geq 0$ for $w_{1} /\left(a_{1} v_{1}\right) \leq 1 / c_{1}$, whence follow the bounds given by (13). Other developments are similar.

The above information immediately leads to the extremal field shown in Figure 5 (see also Section 3.3). 


\subsection{Determination of the Optimal Fire-Support Policy}

The optimality of the extremal fire-support policy developed above follows according to the reasoning given in Section 4.3 by the uniqueness of extremals.

\section{DEVELOPMENT OF OPTIMAL POLICY FOR PROBLEM 3}

In this case we consider (1) with the criterion functional

$$
J_{3}=\left\{\sum_{k=1}^{2} v_{k} x_{k}(T)\right\} /\left\{\sum_{k=1}^{2} w_{k} y_{k}(T)\right\}
$$

\subsection{Necessary Conditions of Optimality}

The necessary conditions of optimality for Problem 3 are the same as those for Problem 2 except that the boundary conditions for the adjoint variables are different. Thus, (55) through (57) also apply to Problem 3. The adjoint system of equations (again using. (2) for convenience) is given by (assuming that $x_{i}(T)>0$ )

where

$$
\begin{gathered}
p_{i}(t)=v_{i} / D \text { for } 0 \leq t \leq T \quad \text { with } i=1,2 \text {, and } \\
\dot{q}_{i}=a_{i} p_{i}+\phi_{i}^{*} c_{i} q_{i} \quad \text { with } q_{i}(T)=-w_{i} J_{3} / D \text { for } i=1,2,
\end{gathered}
$$

$$
D=\sum_{k=1}^{2} w_{k} y_{k}(T)
$$

Computing the first two time derivatives of the switching function

$$
\dot{S}_{\phi}(t)=-a_{1} c_{1} p_{1} y_{1}+a_{2} c_{2} p_{2} y_{2} \text {, and } \ddot{S}_{\phi}(t)=a_{1} c_{1} \dot{p}_{1} y_{1}\left(c_{1} \phi\right)-a_{2} c_{2} p_{2} y_{2}\left(c_{2}(1-\phi)\right),
$$

we find that (60) and (61) again hold on a singular subarc. On such a singular subarc the generalized Legendre-Clebsch condition is satisfied, since

$$
\frac{\partial}{\partial \phi}\left\{\frac{d^{2}}{d t^{2}}\left(\frac{\partial H}{\partial \phi}\right)\right\}=a_{1} c_{1} v_{1} y_{1}\left(c_{1}+c_{2}\right) / D>0 .
$$

\subsection{Synthesis of Extremals}

The synthesis of extremals is essentially the same as for Problem 2 (see Section 5.2 above) except that we have

$$
S_{\phi}(0)=J_{3} a_{2} c_{2} v_{2} y_{2}^{\rho}\left(\frac{w_{1}}{a_{1} v_{1}}\right)\left\{\left(\frac{a_{1} c_{1} v_{1} y_{1}^{\rho}}{a_{2} c_{2} v_{2} y_{2}^{\rho}}\right)-\left(\frac{w_{2}}{a_{2} v_{2}}\right) /\left(\frac{w_{1}}{a_{1} v_{1}}\right)\right\} / D
$$

and

$$
\stackrel{\circ}{S}_{\phi}(\tau)=\left(a_{1} c_{1} v_{1} y_{1}-a_{2} c_{2} v_{2} y_{2}\right) / D
$$

It follows that

$$
S_{\phi}(\tau)=\left\{J_{3} a_{2} c_{2} v_{2} y_{2}^{f}\left(\frac{w_{1}}{a_{1} v_{1}}\right)\left[\left(\frac{a_{1} c_{1} v_{1} y_{1}^{f}}{a_{2} c_{2} v_{2} y_{2}^{f}}\right)-\left(\frac{w_{2}}{a_{2} v_{2}}\right) /\left(\frac{w_{1}}{a_{1} v_{1}}\right)\right]+\int_{c}^{\tau}\left[a_{1} c_{1} v_{1} y_{1}(\sigma)-a_{2} c_{2} v_{2} y_{2}(\sigma)\right] d \sigma\right\} / D .
$$




\subsection{Determination of the Optimal Fire-Support Policy}

As for Problem 2, the optimality of the extremal fire-support policy developed above follows according to the reasoning given in Section 4.3 by the uniqueness of extremals.

\section{DEVELOPMENT OF OPTIMAL POLICY FOR PROBLEM 4}

In this case we consider (1) with the criterion functional

$$
\dot{J}_{4}=-\left\{\sum_{k=1}^{2} v_{k}\left[x_{k}^{0}-x_{k}(T)\right]\right\} /\left\{\sum_{k=1}^{2} w_{k}\left[y_{k}^{0}-y_{k}(T)\right]\right\}
$$

The necessary conditions of optimality for Problem 4 are the same as those for Problems 2 and 3, except that the boundary conditions for the adjoint variables are different: at $t=T$ we have

$$
p_{i}(T)=v_{i} / D_{1} \text { and } q_{i}(T)=-w_{i}\left(-J_{4}\right) / D_{1} \text { for } i=1,2,
$$

where

$$
D_{1}=\sum_{k=1}^{2} w_{k}\left[y_{k}^{0}-y_{k}(T)\right]
$$

Consequently, the solution to Problem 4 is exactly the same as that to Problem 3 , except that $J_{3}$ in the solution to Problem 3 is replaced by $\left(-J_{4}\right)$. Because of the dependence of $J_{4}$ on the initial force levels $x_{i}{ }^{0}, y_{i}{ }^{\circ}$ for $i=1,2$, the two-point boundary-value problem which arises in the determination of switching times when (25) holds is very difficult to solve.

\section{DISCUSSION}

In this section we discuss what we have learned about the dependence of the structure of optimal time-sequential fire-support policies on the quantification of military objectives. We studied this dependence by considering four specific problems (each corresponding to a different quantification of objectives, i.e. criterion functional) for which solutions weresdeveloped by modern optimal control theory.

Our most significant finding is that essentially the entire structure of the optimal fire-support policy may be changed by modifying the quantification of military objectives. We feel that there are basically two types of military strategies: (1) to obtain a "local" advantage, and (2) to obtain an "overall" advantage. The criterion function for Problem 1 (i.e.

$$
J_{1}=\sum_{k=1}^{2} \alpha_{k} x_{k}(T) / y_{k}(T)
$$

a weighting of the final force ratios in the two separate combat areas) reflects the striving to attain a "local" advantage (referred to above as a "breakthrough" tactic). The corresponding optimal fire-support policy was to concentrate all supporting fires on one of the enemy units (the quantitative determination of this policy is given in Section 3.3) for the entire period of fire support. However, we have assumed that the $X$ commander has perfect information about the state variables (e.g. enemy force levels) and all Lanchester attrition-rate coefficients (i.e. system parameters). In the real world where this assumption may not hold, this policy need not be optimal. Other factors 
that would temper the use of such a policy in the real world are (1) the need to "pin down" enemy forces with supporting fires (i.e. suppressive effects), and (2) the giving of information to the enemy as to exactly where his defenses will be attacked by the concentration of preparatory fires only there.

On the other hand, the criterion functionals for Problems 2, 3, and 4 reflect the striving to attain an "overall" advantage (referred to above as an "attrition" tactic which aims to wear down the overall enemy strength). The corresponding optimal fire-support policies for Problems 2, 3 , and 4 were qualitatively the same and could involve a splitting of supporting fires between the two enemy troop concentrations. This property of the optimal fire-distribution policy is not present in the solution to Problem 1 and was anticipated by our earlier work on optimal fire distribution against enemy target types which undergo attrition according to a "linear-law" process (see Section 3.1 above) [31, 32]. The criterion functional for this earlier work was the difference between the overall military worths of friendly and enemy survivors. Thus, we see that nonconcentration of fires on particular target types is characteristic of optimal time-sequential fire distribution over enemy target types which undergo attrition according to a "linear-law" process with the objective of attaining an "overall" advantage.

We saw that the structures of the optimal fire-support policies for Problems 2, 3, and 4 were qualitatively similar. In fact, when one (i.e. the $X$ commander) values enemy (i.e. $Y$ ) forces in each of the two combat zones in direct proportion to their rate (per unit of individual weapon system) of destroying the value of opposing friendly forces, the optimal policies were exactly the same for all three problems (see Section 3.3). In this case the optimal fire-support policy took the particularly simple form of Policy A as given by (6).

When enemy survivors were not valued in direct proportion to their rate of destruction of friendly value, the optimal policies were different and more complex (see Section 3.3, in particular Figure 5), and the planning horizon may be considered to be divided into two phases, denoted as Phase I and Phase II. The lengths of these two phases depended on different factors in these three problems, and the timing of changes in the allocation of supporting fires could be appreciably different. When the planning objective was the maximization of the difference in the total military worths of friendly and enemy forces at the end of the "approach to contact," the length of, for example, Phase II (during which all fire is concentrated on $Y_{1}$ ) depended only on the attrition-rate coefficients and enemy force levels and was independent of the friendly attacking-force levels. When the ratio of the total worths of surviving friendly and enemy forces was considered (i.e. for Problem 3), the length of Phase II also depended directly on the attacking friendly force levels, while when the ratio of the total worths of friendly and enemy losses was considered, it also depended on the initial total worths of forces.

Thus, we see that (at least for the relatively simple fire-support allocation problem considered here) the structure of the optimal time-sequential allocation policy may be strongly influenced by the quantification of military objectives. Moreover, the most important planning decision apparently is whether, a side will seek to attain an "overall" advantage or a "local" advantage. We hope that our investigation has provided a better understanding of the dependence of the structure of optimal fire-support strategies on combatant objectives. In conclusion, it appears to us that more such specific cases warrant investigation for developing a theory of optimal combat strategies. 


\section{APPENDIX}

\section{Fire-Support Allocation Problem in Which $X_{i}$ 's Fire on $Y_{i}$ is Not Neglected}

When $X_{i}$ 's fire effectiveness against $Y_{i}$ is not assumed to be negligible, the fire-support allocation problem (1) considered in the main text becomes

$$
\underset{\phi_{i}(t)}{\operatorname{maximize}} J
$$

with stopping rule: $t_{\rho}-T=0$,

subject to:

(battle dynamics)

$$
\frac{d x_{i}}{d t}=-a_{i} y_{i}
$$

$$
\frac{d y_{i}}{d t}=-b_{i} x_{i}-\phi_{i} c_{i} y_{i} \quad \text { for } i=1,2
$$

with

$$
\begin{aligned}
& x_{1}, x_{2}, y_{1}, y_{2} \geq 0, \\
& \phi_{1}+\phi_{2}=1, \text { and } \phi_{i} \geq 0 \quad \text { for } i=1,2 .
\end{aligned}
$$

Unfortunately, the optimal policy to (A.1), for example, for the criterion functional $J_{1}$ does not take a simple form at all [44]. It appears that without the approximation used in the main text (i.e. $b_{i}=0$ ), it is essentially impossible to analytically develop deep insights into the structure of the optimal policy. See Appendix A in the report by Taylor [44] for further details. Since our goal has been to investigate the dependence of the optimal policy on the quantification of objectives (see also Taylor [37]), we have chosen to study the simpler problems.

\section{REFERENCES}

[1] Anderson, L. B., J. Bracken, J. Falk, J. Grotte, and E. Schwartz, "On the Use of Max-Min and Min-Max Strategies in Multistage Games and ATACM," P-1197, Institute for Defense Analyses, Arlington, Virginia, (August 1976).

[2] Anderson, L. B., J. Bracken, and E. Schwartz, "Revised OPTSA Model," P-1111, Institute for Defense Analyses, Arlington, Virginia, (September 1975).

[3] Antosiewicz, H., "Analytic Study of War Games," Naval Research Logistics Quarterly 2, 181-208 (1955).

[4] Bellman R., and Dreyfus, S., "On a Tactical Air-Warfare Model of Mengel," Operations Research 6,65-78 (1958).

[5] Bracken, J., "Two Optimal Sortie Allocation Models, Volume I: Methodology and Sample Results," P-992, Institute for Defense Analyses, Arlington, Virginia, (December 1973).

[6] Bracken J., J. Falk, and A. Karr, "Two Models for Optimal Allocation of Aircraft Sorties," Operations Research 23, 979-995 (1975).

[7] Brackney, H., "The Dynamics of Military Combat," Operations Research 7, 30-44 (1959).

[8] Bryson, A., and Y. O. Ho, Applied Optimal Control (Blaisdell Publishing Company, Waltham, Massachusetts, (1969).

[9] Chattopadhyay, R., "Differential Game Theoretic Analysis of a Problem of Warfare," Naval Research Logistics Quarterly 16, 435-441 (1969). 
[10] Fish, J., "ATACM:ACDA Tactical Air Campaign Model," ACDA/PAB-249, Ketron, Inc., Arlington, Virginia, (October 1975).

[11] Funk, J., and E. Gilbert, "Some Sufficient Conditions for Optimality in Control Problems with State Space Constraints;" SIAM Journal on Control 8, 498-504 (1970).

[12] Galiano, R., and F. Miercort. "Results of a Survey of Tactical Air Campaign Models," Ketron, Inc., Arlington, Virginia, (November 1974).

[13] Giamboni, L., A. Mengel, and R. Dishington, "Simplified Model of a Symmetric Tactical Air War," The RAND Corporation, RM-711, (August 1951).

[14] Harris, K., and L. Wegner, and "Tactical Airpower in NATO Contingencies: A Joint AirBattle/Ground-Battle Model (TALIY/TOTEM)," The RAND Corporation, R-1194-PR, (May 1974).

[15] Ho, Y. C., "Toward Generalized Control Theory;" IEEE Transactions on Automatic Control, Vol. AC-14, 753-754 (1969).

[16] Ho, Y. C., "Differential Games, Dynamic Optimization, and Generalized Control Theory," Journal of Optimization Theory and Applications 6, 179-209 (1970).

[17] Howes, D., and R. Thrall, "A Theory of Ideal Linear Weights for Heterogeneous Combat Forces," Naval Research Logistics Quarterly 20, 645-659 (1973).

[18] Isaacs, R., Differential Games (John Wiley, New York, 1965).

[19] Karr, A., "Stochastic Attrition Models of Lanchester Type," P-1030, Institute for Defense Analyses, Arlington, Virginia, (June 1974).

[20] Kawara, Y., "An Allocation Problem of Fire Support in Combat as a Differential Game," Operations Research 21, 942-951 (1973).

[21] Kelley, H., R. Kopp, and H. Moyer, "Singular Extremals;" in Topics in Optimization, G. Leitman (Ed.), pp. 63-101 (Academic Press, New York, 1967).

[22] Lansdowne, Z., G. Dantzig, R. Harvey; and R. McKnight, "Development of an Algorithm to Solve Multi-State Games;" Control Analysis Corporation, Palo Alto, California, (May 1973).

[23] Lee, E., and M. Markus, Foundations of Optimal Control Theory, (John Wiley \& Sons, Inc., New York, 1967).

[24] Mangasarian, O., "Sufficient Conditions for the Optimal Control of Nonlinear Systems," SIAM Journal on Control 4, 139-152 (1966).

[25] McNicholas, R., and F. Crane, "Guide to Fire Support Mix Evaluation Techniques, Volume I: The Guide and Appendices A and B." Stanford Research Institute, Menlo Park, California, (March 1973).

[26] Moglewer, S., and C. Payne, "A Game Theory Approach to Logistics Allocation," Naval Research Logistics Quarterly 17, 87-97 (1970).

[27] Morse, P., and G. Kimball, Methods of Operations Research, (The M.I.T. Press, Cambridge, Massachusetts, 1951).

[28] Pontryagin, L., V. Boltyanskii, R. Gamkrelidze, and E. Mișhchenko, The Mathematical Theory of Optimal Processes, (Interscience, New York, 1962).

[29] Pugh, G., and J. Mayberry, "Theory of Measures of Effectiveness for General-Purpose Military Forces: Part I. A Zero-Sum Payoff Appropriate for Evaluating Combat Strategies," Operations Research 21, 867-885 (1973). 
[30] Taylor, J., "On the Isbell and Marlow Fire Programming Problem," Naval Research Logistics Quarterly 19, 539-556 (1972).

[31] Taylor, J., "Lanchester-Type Models of Warfare and Optimal Control," Naval Research Logistics Quarterly 21, 79-106 (1974).

[32] Taylor, J., "Target Selection in Lanchester Combat: Linear-Law Attrition Process," Naval Research Logistics Quarterly 20, 673-697 (1973).

[33] Taylor, J., "Target Selection in Lanchester Combat: Heterogeneous Forces and Time-Dependent Attrition-Rate Coefficients," Naval Research Logistics Quarterly 21, 683-704 (1974).

[34] Taylor, J., "Solving Lanchester-Type Equations for 'Modern Warfare' with Variable Coefficients," Operations Research 2Q, 756-770 (1974).

[35] Taylor, J., "On the Treatment of Force-Level Constraints in Time-Sequential Combat Problems," Naval Rseearch Logistics Quarterly 22, 617-650 (1975).

[36] Taylor, J. "On the Relationship Between the Force Ratio and the Instantaneous CasualtyExchange Ratio for Some Lanchester-Type Models of Warfare," Naval Research Logistics Quarterly 23, 345-352 (1976).

[37] Taylor, J., "Determining the Class of Payoffs that Yield Force-Level-Independent Optimal Fire-Support Strategies," Operations Research 25, 506-516 (1977).

[38] Taylor, J., and G. Brown, "Canonical Methods in the Solution of Variable-Coefficient Lanchester-Type Equations of Modern Warfare," Operations Research 24, 44-69 (1976).

[39] Taylor, J., and S. Parry, "Force-Ratio Considerations for Some Lanchester-Type Models of Warfare," Operations Research 23, 522-533 (1975).

[40] Taylor, J., "Survey on the Optimal Control of Lanchester-Type Attrition Processes," presented at the Symposium on the State-of-the-Art of Mathematics in Combat Models, (June 1973) (also Tech. Report NPS55Tw74031, Naval Postgraduate School, Monterey, California, March 1974) (AD 778 630).

[41] Taylor, J., "Application of Differential Games to Problems of Military Conflict: Tactical Allocation Problems-Part II," Naval Postgraduate School Tech. Report No. NPS55Tw7211A. Monterey, California, (November 1972) (AD 758663 ).

[42] Taylor, J., "Application of Differential Games to Problems of Military Conflict: Tactical Allocation Problems-Part III," Naval Postgraduate School Tech. Report No. NPS55Tw74051, Monterey, California, (May 1974) (AD 782 304).

[43] Taylor, J., "Appendices C and D of 'Application of Differential Games to Problems of Military Conflict: Tactical Allocation Problems-Part III"," Naval Postgraduate School Tech. Report No. NPS55Tw74112, Monterey, California, (November 1974) (AD A005 872).

[44] Taylor, J., "Optimal Fire-Support Strategies," Naval Postgraduate School Tech. Report No. NPS55Tw76021, Monterey, California, (February 1976) (AD A033 761).

[45] Taylor, J., and G. Brown, "An Examination of the Effects of the Criterion Functional on Optimal Fire-Support Policies," Naval Postgraduate School Tech. Report No. NPS55Tw76092, Monterey, California, (September 1976) (AD A033 760).

[46] USAF Assistant Chief of Staff, Studies and Analysis, "Methodology for Use in Measuring the Effectiveness of General Purpose Forces, SABER GRAND (ALPHA)," (March 1971).

[47] Weiss, H., "Lanchester-Type Models of Warfare," in Proc. First International Conf. Operational Research pp. 82-98 (John'Wiley \& Sons, Inc., New York, 1957).

[48] Weiss, H., "Some Differential Games of Tactical Interest and the Value of a Supporting Weapon System," Operations Research 7, 180-196 (1959). 